\title{
Differential Expression of Fungal Genes Determines the Lifestyle of Plectosphaerella Strains During Arabidopsis thaliana Colonization
}

\author{
Antonio Muñoz-Barrios, ${ }^{1,2}$ Sara Sopeña-Torres, ${ }^{1}$ Brisa Ramos, ${ }^{1}$ Gemma López, ${ }^{1}$ Irene del Hierro, ${ }^{1,2}$ \\ Sandra Díaz-González,, ${ }^{1,2}$ Pablo González-Melendi, ${ }^{1,2}$ Hugo Mélida, ${ }^{1}$ Vanessa Fernández-Calleja, ${ }^{1}$ \\ Verónica Mixão, ${ }^{3,4}$ Marina Martín-Dacal, ${ }^{1,2}$ Marina Marcet-Houben,, ${ }^{3,4}$ Toni Gabaldón,, ${ }^{3,4,5}$ \\ Soledad Sacristán, ${ }^{1,2,+}$ and Antonio Molina ${ }^{1,2,+}$ \\ ${ }^{1}$ Centro de Biotecnología y Genómica de Plantas, Universidad Politécnica de Madrid (UPM)-Instituto Nacional de Investigación \\ y Tecnología Agraria y Alimentaria (INIA), Campus Montegancedo-UPM, 28223-Pozuelo de Alarcón (Madrid), Spain \\ ${ }^{2}$ Departamento de Biotecnología-Biología Vegetal, Escuela Técnica Superior de Ingeniería Agronómica, Alimentaria y de \\ Biosistemas, Universidad Politécnica de Madrid, 28040-Madrid, Spain \\ ${ }^{3}$ Centre for Genomic Regulation (CRG), Barcelona Institute of Science and Technology, Dr. Aiguader 88, 08003 Barcelona, \\ Spain \\ ${ }^{4}$ Universitat Pompeu Fabra (UPF), 08003 Barcelona, Spain \\ ${ }^{5}$ ICREA, Pg. Lluís Companys 23, 08010 Barcelona, Spain
}

Accepted 25 July 2020.

The Whole-Genome Shotgun projects have been deposited in the DDBJ/ENA/GenBank database under accessions JACAFV000000000 (Plectosphaerella sp. strain P0831 Biosample SAMN14235337 TaxID: 40657), JACAFW000000000 (P. cucumerina Pc2127 Biosample SAMN14233823 TaxID: 40658), and JACAFX000000000 P. cucumerina (PcBMM Biosample SAMN14233534) TaxID: 40658, and BioProject number PRJNA609142. The versions described by this paper are versions JACAFV010000000, JACAFW010000000 and JACAFX010000000. The raw RNA-seq data in this study are deposited in the National Center for Biotechnology Information Sequence Read Archive (SRA), BioProject number PRJNA614936 and BioSamples SAMN14444085 to SAMN14444096, SAMN14444149, SAMN14444167, and SAMN14444168, with SRA accessions SRR11668188 to SRR11668202.

Current address for Toni Gabaldón: Barcelona Supercomputing Centre and Institute for Research in Biomedicine, Jordi Girona 29, 08034 Barcelona, Spain

${ }^{\dagger}$ Corresponding authors: S. Sacristán; soledad.sacristan@upm.es and A. Molina; antonio.molina@upm.es

Funding: This work was supported by the Spanish Ministry of Economy and Competitiveness (MINECO) grant BIO2015-64077-R and the Spanish Research Agency (AEI) grant RTI2018-096975-B-I00 to A. Molina and by the "Severo Ochoa Programme for Centers of Excellence in R\&D" grant SEV-2016-0672 (2017-2021) to the CBGP (UPM-INIA). In the frame of SEV-2016-0672 program, H. Mélida was supported with a postdoctoral contract. A. Muñoz-Barrios was financially supported by the Universidad Politécnica de Madrid (UPM) Ph.D. students PIF program, I. del Hierro was a FPU fellow (Spanish Ministry of Education, Culture and Sports grant FPU16/07118), V. Fernández-Calleja was supported by the Consejería de Educacíon e Investigacíon of Comunidad de Madrid YEI program for postdoctoral researchers (PEJD-2016/BIO-3327), and the work was further supported through a Comunidad de Madrid YEI program for laboratory technicians grant (PEJ16/BIO/TL-1570).

*The $\boldsymbol{e}$-Xtra logo stands for "electronic extra" and indicates there are supplementary figures and tables published online.

The author(s) declare no conflict of interest.

๑) 2020 The American Phytopathological Society
The fungal genus Plectosphaerella comprises species and strains with different lifestyles on plants, such as $P$. cucumerina, which has served as model for the characterization of Arabidopsis thaliana basal and nonhost resistance to necrotrophic fungi. We have sequenced, annotated, and compared the genomes and transcriptomes of three Plectosphaerella strains with different lifestyles on $A$. thaliana, namely, PcBMM, a natural pathogen of wild-type plants (Col-0), Pc2127, a nonpathogenic strain on Col-0 but pathogenic on the immunocompromised cyp79B2 cyp79B3 mutant, and P0831, which was isolated from a natural population of $A$. thaliana and is shown here to be nonpathogenic and to grow epiphytically on Col-0 and cyp79B2 cyp79B3 plants. The genomes of these Plectosphaerella strains are very similar and do not differ in the number of genes with pathogenesis-related functions, with the exception of secreted carbohydrate-active enzymes (CAZymes), which are up to five times more abundant in the pathogenic strain PcBMM. Analysis of the fungal transcriptomes in inoculated Col-0 and cyp79B2 cyp79B3 plants at initial colonization stages confirm the key role of secreted CAZymes in the necrotrophic interaction, since PcBMM expresses more genes encoding secreted CAZymes than Pc2127 and P0831. We also show that P0831 epiphytic growth on $A$. thaliana involves the transcription of specific repertoires of fungal genes, which might be necessary for epiphytic growth adaptation. Overall, these results suggest that in-planta expression of specific sets of fungal genes at early stages of colonization determine the diverse lifestyles and pathogenicity of Plectosphaerella strains.

Keywords: Arabidopsis, CAZyme, epiphytic fungus, genome, immunity, pathogenicity, necrotroph, Plectosphaerella

Plants are continuously encountering a diverse array of microorganisms that differ in their ability to infect and cause disease (Brader et al. 2017; Newton et al. 2010; Zeilinger et al. 2016). Microbial pathogens have genetic repertoires required for the colonization of plant tissues and molecular tools to overcome plant immune responses (Rai and Agarkar 2016). 
Nonpathogenic microorganisms may be able to survive on host surfaces, growing epiphytically and establishing specific types of interactions with their hosts (Leveau 2015; Lindow and Brandl 2003; Vorholt 2012; Whipps et al. 2008). Little is known about the genetic mechanisms underlying the epiphytic interactions of fungi with plants and their differences with pathogenic fungi, and similarly, our knowledge about the function of plant immunity in these epiphytic interactions is scarce (Rastogi et al. 2013; Vorholt 2012; Yao et al. 2019). However, fungal epiphytes represent a considerable fraction of the phyllosphere microbiome that can greatly affect plant fitness, either positively or negatively (Hongsanan et al. 2016; Vorholt 2012; Whipps et al. 2008). For example, it has been shown that fungal epiphytes can protect plants against foliar pathogens, either as microbial antagonists (Zhou et al. 2017) or by priming plant resistance (Buxdorf et al. 2013). On the other hand, epiphytic growth could be an important phase in the interaction with the plant prior to infection or could be used as a survival mechanism in the absence of a suitable host (Vorholt 2012). Comparing pathogenic and nonpathogenic plant-fungus interactions is an excellent strategy to find molecular determinants of pathogenicity and virulence, as shown by several recent studies (Baetsen-Young et al. 2020; Hacquard et al. 2016; Plett and Martin 2018; Zeilinger et al. 2016). Since the nature of nonpathogenic interactions is diverse, additional comparative studies are needed in other fungus-plant interactions to better understand the genetic bases of nonpathogenic lifestyles of fungi.

Fungal plant pathogens are classified as biotrophs, hemibiotrophs, and necrotrophs, each having different modes of interaction with their host plants (Horbach et al. 2011). Biotrophic pathogens are usually obligate parasites that do not kill host cells and establish sophisticated interactions with the host that include the secretion of effectors that manipulate the plant metabolism, suppress the immune responses, and promote the trophic interaction (Spanu and Panstruga 2017). Necrotrophic pathogens rapidly cause substantial tissue damage, killing host cells by a combination of mechanisms involving, among others, the expression of cell wall-degrading enzymes (CWDEs) that hydrolyze plant cell-wall polymers, the production of reactive oxygen species (ROS) and the secretion of toxins (Wang et al. 2014). These activities lead to cell wall and membrane disruption in the host cells and to the release of nutrients, which favor extensive colonization of a plant host by the fungus and tissue decomposition (Zeilinger et al. 2016). Despite being less-studied than biotrophic interactions, increasing evidence shows that the molecular mechanisms of plant and necrotrophic fungi interactions are complex and involve fungal effectors (Wang et al. 2014).

The Ascomycete genus Plectosphaerella includes several species commonly found in the rhizosphere and strains isolated from very different hosts, mainly plants, but also from insects, crustaceans, or nematodes (Giraldo and Crous 2019; Yu and Coosemans 1998). Pathogenic Plectosphaerella spp. have been widely reported to cause fruit, root, and collar rot on several crops, becoming an emergent pathogen in recent decades (Carlucci et al. 2012; Dillard et al. 2005; Jimenez and Zitter 2005; Su et al. 2017; Usami and Katagiri 2017; Vitale et al. 2007). However, Plectosphaerella species can also exhibit other lifestyles in plants, as endophytes colonizing plant tissues without causing visible symptoms (D'Amico et al. 2008; Götz et al. 2006) or as epiphytes showing antagonistic effects against bacterial pathogens (Zhou et al. 2017). Plectosphaerella strains have also been found in natural populations of Arabidopsis thaliana, either in pathogenic associations (Durán et al. 2018; Ton and Mauch-Mani 2004) or as endophytes in asymptomatic plants (García et al. 2013; Junker et al. 2012; Thiergart et al. 2020).

The interaction between Arabidopsis and the species $P$. cucumerina is a well-established pathosystem for the study of plant basal and nonhost resistance to necrotrophic fungi (Ramos et al. 2013; Sánchez-Vallet et al. 2010). The analysis of this pathosystem has contributed to the identification of novel components of plant defense mechanisms. For example, it has been found that the biosynthesis of tryptophan (Trp)-derived metabolites (depleted in cyp79B2 cyp79B3, pen2, cyp81f2, and cyp71A12 mutants) and their targeted delivery at pathogen contact sites (impaired in pen3 mutant) are required for Arabidopsis basal resistance to both pathogenic (e.g., PcBMM) and nonpathogenic strains (e.g., Pc2127) of P. cucumerina (Bednarek et al. 2009; Hernández-Blanco et al. 2007; Lipka et al. 2005; Pastorczyk et al. 2020; Sánchez-Vallet et al. 2010; Stein et al. 2006). Additional signaling pathways are also involved in Arabidopsis resistance to the pathogenic strain PcBMM, like those mediated by the defense hormones salicylic acid (SA), jasmonate (JA), ethylene (ET), abscisic acid (ABA), and cytokinin, by heterotrimeric $\mathrm{G}$ protein and ERECTA receptor like kinase, or by signaling mechanisms triggered upon alteration of plant cell-wall integrity (Bacete et al. 2020; Berrocal-Lobo et al. 2002; Delgado-Cerezo et al. 2012; Hernández-Blanco et al. 2007; Llorente et al. 2005, 2008; Sánchez-Vallet et al. 2012). Also, immune responses triggered by microbe-associated molecular patterns (MAMPs) are required for Arabidopsis resistance to $P$. cucumerina. The cell walls of spores and mycelium of PcBMM contain several glycans, like chitin and 1,3$\beta$-glucans, that are perceived as MAMPs by plant pattern recognition receptors (PRRs) like CERK1, triggering transcriptional regulation of immune-related genes (Bacete et al. 2018; Mélida et al. 2018). Accordingly, Arabidopsis cerkl mutant is immunocompromised and shows enhanced susceptibility to $P$. cucumerina and to other fungi and oomycetes (Mélida et al. 2018; Wan et al. 2008).

To characterize the genetic determinants of the interactions between Plectosphaerella spp. and Arabidopsis, we have sequenced, annotated, and compared the genomes and transcriptomes of three fungal strains with different lifestyles (PcBMM, Pc2127, and P0831). PcBMM was isolated from Arabidopsis and is an adapted necrotrophic pathogen in all Arabidopsis genotypes tested, whereas the nonadapted Pc2127, isolated from a different host, is unable to colonize Arabidopsis wild-type plants (Col-0) but is pathogenic on immunocompromised double mutant cyp79B2 cyp79B3 plants (Bednarek et al. 2009; Sánchez-Vallet et al. 2010). Notably, we show here that strain P0831, isolated from a leaf of an asymptomatic Arabidopsis plant from a natural population in central Spain (García et al. 2013), is nonpathogenic on Col-0 and cyp79B2 cyp79B3 plants but is able to grow epiphytically on leaves of these genotypes. Here, we describe the whole sequencing and characterization of the genomes of these strains, showing that they have minor differences in the number of genes encoding pathogenesis-related functions. Our data point to secreted carbohydrate-active enzymes (CAZymes) as one of the main pathogenicity determinants of strain PcBMM. Our analyses also show that there are significant differences in the number and predicted function of fungal genes expressed in the interaction of the epiphytic P0831 strain with Arabidopsis plants compared with those established by the adapted and nonadapted strains.

\section{RESULTS}

\section{Plectosphaerella strains display different lifestyles in Arabidopsis leaves.}

We collected asymptomatic Arabidopsis plants from natural populations in central Spain and, by PCR, detected the genus Plectosphaerella in up to 50 and $27 \%$ of their leaves and roots, respectively (Supplementary Fig. S1). Plectosphaerella P0831 strain was isolated from the leaf of one of these asymptomatic 
plants (García et al. 2013). The growth and pathogenicity patterns of strain P0831 on Arabidopsis Col-0 leaves were analyzed and compared with those of the well-characterized PcBMM and Pc2127 strains, which are pathogenic and nonpathogenic in Col-0 plants, respectively (Sánchez-Vallet et al. 2010; Ramos et al. 2013). P0831, like Pc2127, did not produce any disease symptoms on Col-0 plants, whereas PcBMM caused necrotic spots on leaves that spread and reached the vascular system, as previously described (Fig. 1A) (Ramos et al. 2013). By using fungal transformants of the three strains constitutively expressing the green fluorescence protein (GFP) (PcBMM-GFP and Pc2127-GFP [Ramos et al. 2013] and P0831-GFP [generated in this work]), we found that P0831GFP spores germinated and formed a dense mycelium on Col-0 leaf surfaces that could be observed at 4 days postinoculation (dpi), whereas Pc2127 spores germinated on Col-0 leaf surfaces but did not form mycelia, as previously reported (Fig. 1B) (Ramos et al. 2013). Of note, besides the epiphytic growth of P0831-GFP, hyphae of P0831-GFP were observed in the intercellular space at the mesophyll at 4 dpi (Supplementary Fig. S2). Thus, we concluded that P0831 is not pathogenic on Col-0 leaves, being able to grow epiphytically and, occasionally, endophytically on this accession. Since the pathogenic PcBMM strain was also isolated from Arabidopsis plants and several Plectosphaerella sp. strains have recently been identified in the characterization of Arabidopsis fungal microbiomes (Durán et al. 2018; García et al. 2013; Junker et al. 2012; Thiergart et al. 2020; Ton and Mauch-Mani 2004), we can conclude that the genus Plectosphaerella can display different lifestyles on natural populations of Arabidopsis.

We next tested the interaction of P0831 with the Arabidopsis immunocompromised double mutant cyp79B2 cyp $79 B 3$ that is depleted of all Trp-derived secondary metabolites required for basal resistance (Bednarek et al. 2009; Sánchez-Vallet et al. 2010). Notably, we found that P0831 also grew epiphytically, in this double mutant, without causing disease symptoms (Fig. 1A and B). This contrasted with the previously described lifestyle of the nonpathogenic strain Pc2127 that, like PcBMM, colonized and caused disease symptoms in cyp79B2 cyp79B3 plants, as determined by quantitative PCR (qPCR) of the fungal $\beta$-tubulin gene (Fig. 1A and C) (Sánchez-Vallet et al. 2010). The slight growth of $\mathrm{P} 0831$ in cyp79B2 cyp79B3 was further corroborated at different days postinoculation by trypan blue (TB) staining of inoculated leaves, which revealed that cyp79B2 cyp $79 B 3$ leaves showed a very faint and localized TB staining, suggesting that necrosis was limited to a few plant cells. This contrasted with the intense TB stain, indicative of plant cell death, caused by Pc2127 and PcBMM strains (Supplementary Fig. S3). We also analyzed plant defense responses by determining the production of plant ROS upon fungal inoculation through diaminobenzidine (DAB) staining. We found a weak DAB stain in cyp79B2 cyp79B3 leaves inoculated with Pc2127 and P0831, whereas no DAB staining was observed in Col-0 plants inoculated with P0831 (Supplementary Fig. S3). On the other hand, strong DAB stains were observed in Col-0 and cyp79B2 cyp79B3 plants inoculated with PcBMM, which were indicative of an intense immune response triggered by the progression of fungal growth on leaves (Supplementary Fig. S3).

We next determined whether Arabidopsis plants were able to perceive the three Plectosphaerella strains and to activate immune responses such as the transcriptional upregulation of the RbohD gene, which encodes NADPH oxidase RBHOD involved in ROS production and that has been described to be upregulated by pathogen infection (e.g., PcBMM) or MAMP treatment (Mélida et al. 2018; Morales et al. 2016). Arabidopsis pRbohD::LUC lines (in Col-0 background) expressing the luciferase $(L U C)$ gene under the control of RBOHD promoter $(p R b o h D)$ were inoculated with the fungal strains, and we followed, in vivo, transcriptional regulation of RbohD by determining LUC bioluminescence. We found that bioluminescence at 3 dpi in P0831-inoculated plants was higher than in Pc2127-inoculated plants but lower than the bioluminescence observed in plants inoculated with PcBMM (Supplementary Fig. S4). Notably, LUC bioluminescence of P0831 in Col-0 was also stronger than that caused by the endophytic fungus Colletotrichum tofieldiae 0861 (Ct0861), which was also isolated from a natural Arabidopsis population in central Spain but is unable to grow on the leaf surface (García et al. 2013; Hiruma et al. 2016). These results indicated that Col-0 plants harbor immune mechanisms for the perception of the three Plectosphaerella strains (e.g., PRRs recognizing fungal MAMPs) and the transcriptional upregulation of genes associated with pathogen-associated molecular patterntriggered immunity (PTI).

To further characterize the relevance of Arabidopsis defense mechanisms in shaping Plectosphaerella lifestyle, we tested the colonization of an Arabidopsis ein2 pad4 sid2 dde2 quadruple mutant, defective in all three major phytohormone-dependent defense signaling pathways (ET, SA, and JA) and the effector triggered immunity (ETI) key regulator PAD4 (Mine et al. 2018). Fungal biomass, determined by $\mathrm{qPCR}$ of the $\beta$-tubulin fungal gene, in the ein 2 pad4 sid2 dde 2 mutant inoculated with either Pc2127 or P0831 was similar to that of Col-0 plants, which did not support fungal infection, whereas PcBMM growth in the mutant was higher than in Col-0 plants (Fig. 1C) (Berrocal-Lobo et al. 2002; Lipka et al. 2005; Sánchez-Vallet et al. 2010). Overall, these results confirm the central role of SA, ET, JA, and PAD4-mediated signaling pathways and Trp-derived metabolites in Arabidopsis basal resistance to the pathogenic PcBMM strain and corroborate that Trp-derived basal resistance is sufficient to limit the growth of the nonadapted Pc2127 strain (Sánchez-Vallet et al. 2010). Since impairing SA, ET, JA, and PAD4 signaling pathways or Trp-derived basal resistance does not affect P0831 lifestyle and colonization of Arabidopsis plants, different immune mechanisms may be acting to limit the growth and potential virulence of P0831 epiphytic strain.

\section{Genomic features and phylogenetic relationships of Plectosphaerella strains.}

High-molecular-weight genomic DNA was extracted from mycelia of the three Plectosphaerella strains grown in minimal media. This DNA was used for Illumina single-end (SE), pairedend (PE), and PacBio read-sequencing analyses, which produced sequences covering each fungal genome that were used for de novo genome assembly (Table 1). Plectosphaerella genomes sizes were estimated to be between 37.7 (PcBMM) and 35.9 (Pc2127) Mb (Table 1), which are comparable with the genome sizes of other ascomycete fungi reported (Supplementary Table $\mathrm{S} 1)$. Gene models were predicted, using the Maker v2.31.10 (Cantarel et al. 2008) pipeline, that identified 11,323 (PcBMM), 11,007 (Pc2127) and 10,821 (P0831) genes in the fungal strains (Table 1). Next, we determined the gene space coverage, using BUSCO v3 (Simão et al. 2015), which indicated that 97.5 to $97.7 \%$ of the core conserved genes of the Sordariomycetes database were in the assembled genomes (Table 1).

We used a read mapping approach to compare Pc2127 and P0831 with PcBMM, and we detected the presence of 179,658 single nucleotide polymorphisms (SNPs) (163,473 exclusive) in Pc2127 and 812,707 SNPs (796,522 exclusive) in P0831. In the case of Pc2127, 94\% of the proteins had more than $90 \%$ of the positions with reads in PcBMM and fewer than $0.2 \%$ of the proteins had no reads, which may correspond to putative gene deletions in Pc2127 with respect to PcBMM. P0831 had only $88 \%$ identity at the protein level with PcBMM, which contrasted with $98.6 \%$ identity found for Pc2127, suggesting that 


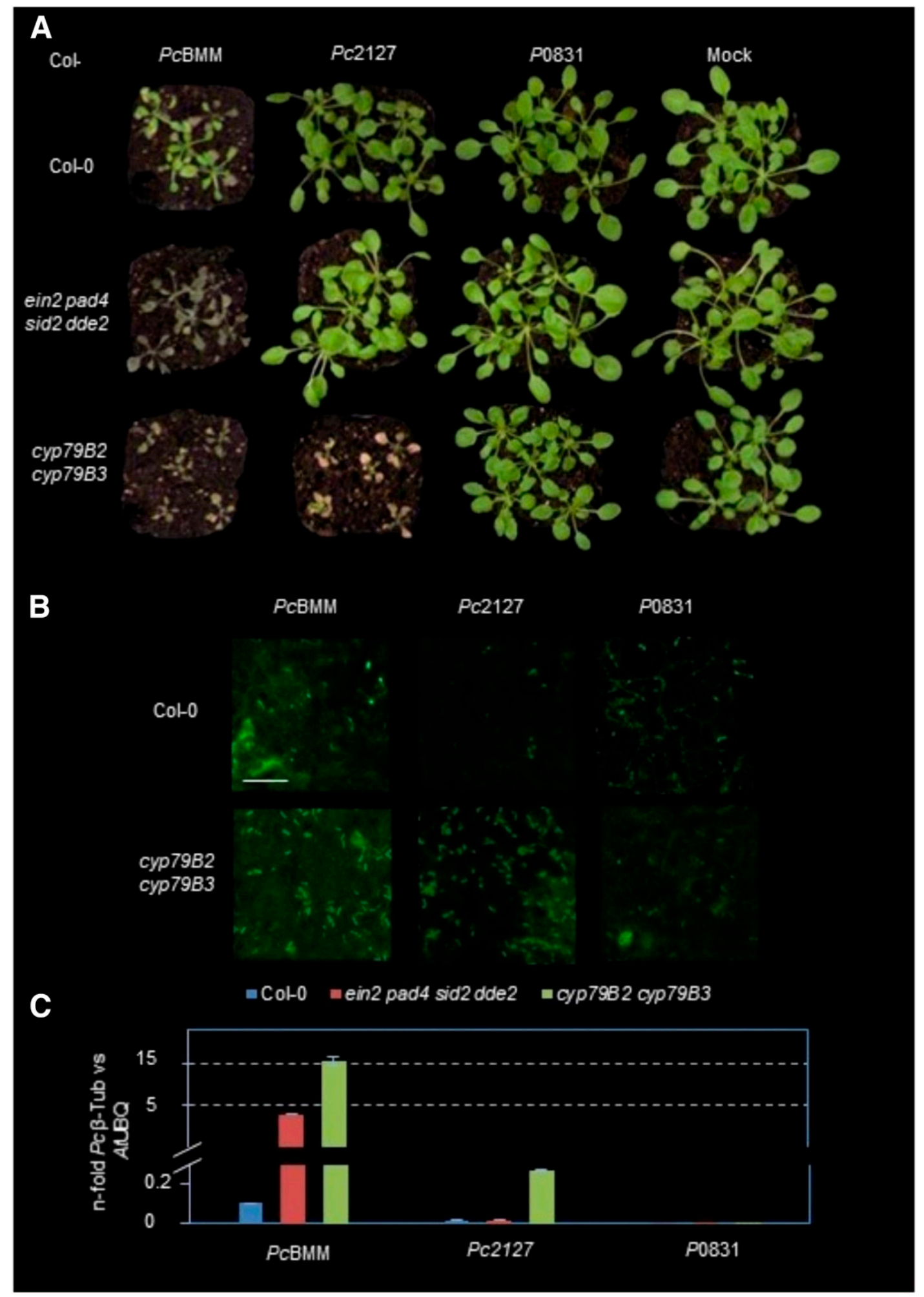

Fig. 1. Differential interactions of PcBMM, Pc2127, and P0831 with Arabidopsis wild-type plants (Col-0) and immune-deficient mutants. Three-week-old Col0 wild-type plants and ein 2 pad 4 sid 2 dde 2 and cyp $79 B 2$ cyp $79 B 3$ mutants were spray-inoculated with spore suspensions $\left(4 \times 10^{6}\right.$ spores per milliliter $)$ of either PcBMM, Pc2127, and P0831 strains or fungal transformants (PcBMM-GFP, Pc2127-GFP, and P0831-GFP) or with water (mock). A, Macroscopic disease symptoms caused in the inoculated plants by PcBMM, Pc2127, and P0831 strains at 7 days postinoculation (dpi). B, Confocal microscopy maximum projections of PcBMM-GFP, Pc2127-GFP, and P0831-GFP spores and mycelium on leaves of wild-type plants (Col-0) and cyp79B2 cyp79B3 mutants at 4 dpi. Scale bar $=50 \mu \mathrm{m}$. C, Quantification of Plectosphaerella cucumerina $\beta$-tubulin DNA in inoculated plants at 3 dpi by quantitative PCR. Values are represented as the average ( \pm standard deviation) of the $n$-fold fungal DNA levels relative to plant ubiquitin (At-UBQ) gene. These experiments were repeated at least three times with similar results. 
P0831 can be considered a different species than PcBMM, while Pc2127 may represent a divergent strain of $P$. cucumerina. Multilocus phylogenetic analysis of the TUB, EF1, and ITS sequences grouped PcBMM and Pc2127 with other members of $P$. cucumerina, whereas P0831 was located outside the clade formed by $P$. cucumerina and $P$. plurivora, further confirming that the P0831 isolate belongs to a different species (Supplementary Fig. S5).

We used OrthoFinder (Emms and Kelly 2015) to cluster protein-coding sequences into sets of homologous genes, comparing Plectosphaerella genomes with those of 24 other fungal species, and these data were used to reconstruct the evolution of every single gene (Fig. 2A; Supplementary Table $\mathrm{S} 1$ ). The phylogenomic analysis confirmed the taxonomic position of the genus Plectosphaerella within the family Plectosphaerelaceae, together with the genus Verticillium, and its proximity to other hemibiotrophic and endophytic species, such as those of the genus Colletotrichum (order Glomerellales), rather than to other necrotrophic fungi such as Botrytis spp. or Sclerotinia spp. (Fig. 2A). The OrthoFinder tool clustered the protein-coding sequences of the three Plectosphaerella isolates in a core of 9,001 gene families that contained orthologs in the three genomes (Fig. 2B), with an average number of 3.3 genes per family (e.g., one ortholog per genome in most of the families). Only a few gene families were exclusive of PcBMM (3), Pc2127 (3), and P0831 (2), accounting, respectively, for 27, 24 , and 27 genes that encoded putative proteins of unknown functions (Fig. 2B). Strain P0831 contained more genes without family (487) assigned by OrthoFinder, followed by PcBMM (412) and Pc2127 (176) (Table 1).

A phylome was reconstructed (stored in PhylomeDB [Huerta-Cepas et al. 2014]) using the proteomes of the three

Table 1. Genome sequencing and assembly statistics of Plectosphaerella strains

\begin{tabular}{|c|c|c|c|}
\hline Parameter & PcBMM & Pc2127 & P0831 \\
\hline Illumina single-end coverage & 26.3 & 27.6 & \\
\hline Illumina paired-end coverage & 27.4 & 28.6 & 27.6 \\
\hline PacBio coverage & 14.6 & & \\
\hline Assembly size & $37.7 \mathrm{Mb}$ & $35.9 \mathrm{Mb}$ & $37.5 \mathrm{Mb}$ \\
\hline No. of contigs $(>1 \mathrm{~kb})$ & 467 & 243 & 388 \\
\hline Largest contig & $1.36 \mathrm{Mb}$ & $0.85 \mathrm{Mb}$ & $0.79 \mathrm{Mb}$ \\
\hline $\mathrm{GC} \%$ & 58.17 & 58.33 & 57.23 \\
\hline N50 length & $338 \mathrm{~kb}$ & $315 \mathrm{~kb}$ & $191 \mathrm{~kb}$ \\
\hline L50 & 36 & 41 & 56 \\
\hline N75 length & $160 \mathrm{~kb}$ & $158 \mathrm{~kb}$ & $107 \mathrm{~kb}$ \\
\hline L75 & 76 & 86 & 122 \\
\hline No. of 'N's in assembly per $100 \mathrm{kbp}$ & 1,054 & 113 & 1,910 \\
\hline No. of predicted gene models & 11,323 & 11,007 & 10,821 \\
\hline Gene space coverage (BUSCO) ${ }^{a}$ & 94.0 to $97.7 \%$ & 92.1 to $97.7 \%$ & 93.2 to $97.5 \%$ \\
\hline No. of genes in orthogroups & 10,911 & 10,831 & 10,333 \\
\hline No. of orthogroups & 9,786 & 9,777 & 9,232 \\
\hline No. of unassigned genes ${ }^{\mathrm{b}}$ & $411(3.6 \%)$ & $176(1.6 \%)$ & $488(4.5 \%)$ \\
\hline
\end{tabular}

a The two numbers indicate fully present and partially present genes.

${ }^{b}$ Percentage of unassigned genes to orthogroups was calculated over total predicted genes.

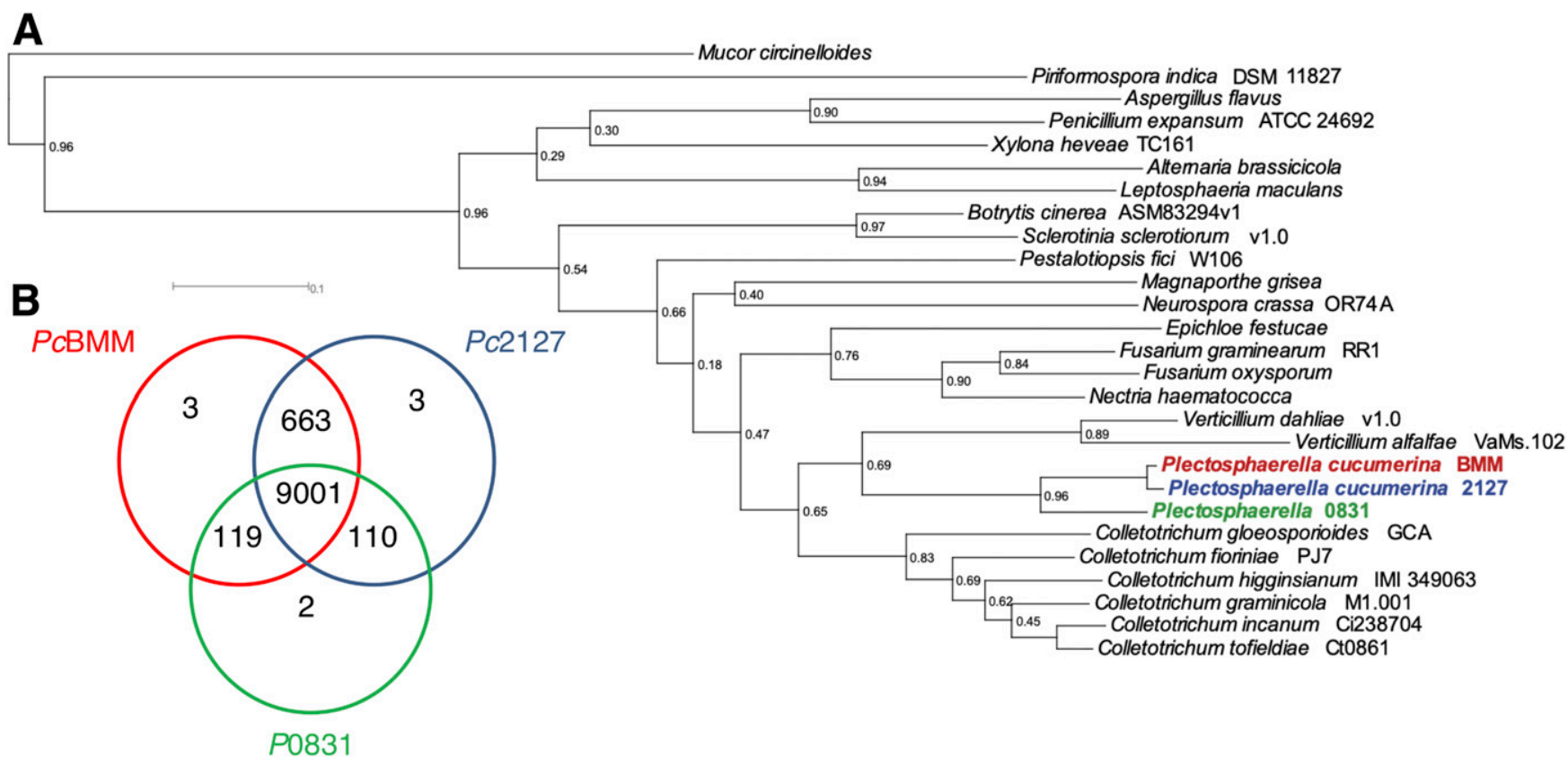

Fig. 2. Genomic relationships of PcBMM, Pc2127, and P0831 genomes with those of other fungi. A, Whole-genome phylogeny inferred by using the STAG method (Emms and Kelly 2018) with Orthofinder algorithm (Emms and Kelly 2015) within each orthogroup of genes present in all 27 compared genomes. A greedy consensus tree was achieved from all these individual estimates to get the final tree. B, Venn diagram distribution of the orthogroup genes generated by comparison of the three Plectosphaerella strains using Orthofinder algorithm. 
Plectosphaerella strains and those of the 24 fungal species listed in Supplementary Table S1. Analysis to identify acquired genes in the genome of PcBMM allowed us to identify 159 orphan genes in PcBMM, 23 gained genes in the first node (shared by PcBMM and Pc2127), and 296 gained genes in the second one (shared by the three strains). Losses were also explored, and we identified 533 genes lost by Pc2127, and 1,112 genes lost by P0831 (Supplementary Fig. S6). For all the acquired genes, a BLASTp (Zhang et al. 2000) search against the UniProt database was performed, finding 10 putative cases of horizontal gene transfers corresponding to fungal genes that had homology with bacterial proteins (Supplementary Table S2). The percentage of identity between these pairs of homologous genes was very low (between 20 and 40\%), suggesting they are not due to a contamination. Moreover, to find whether gained genes were enriched in any particular function, an enrichment analysis with FatiGO (Al-Shahrour et al. 2004) was performed for each of the referred nodes, but no enrichment was found in any of those analyses.

\section{Genes encoding putative secreted CAZYmes are overrepresented among PcBMM pathogenicity and virulence-related genes.}

We looked for predicted functions (proteins) related to pathogenesis in the three Plectosphaerella genomes by determining CAZymes, secreted proteins (secretome), membrane-associated transporters, and genes associated with secondary metabolism (Table 2). The proportions of these pathogenesis-related groups were practically identical among the three genomes, indicating that the genomic structure linked to known pathogenic mechanisms did not differ significantly among the three Plectosphaerella strains. However, we found within the secretome that the predicted secreted CAZymes were up to five times more abundant in the pathogenic PcBMM strain than in Pc2127 and P0831 (Table 2; Supplementary Table S3). Around $75 \%$ of the predicted secreted CAZymes of PcBMM had modules corresponding to CWDEs, such as carbohydrate esterases (CE), glycoside hydrolases (GH), and polysaccharide lyases (PL), that can potentially hydrolyze all the major plant cell-wall polysaccharides (e.g., pectin, hemicellulose, and cellulose [Supplementary Table S3]). Remarkably, secreted carbohydrate-binding modules (CBMs), which have functions related to adhesion to carbohydrates, and secreted auxiliary enzymes with redox activities (AA) were also highly enriched in PcBMM in comparison with Pc2127 and P0831. On the other hand, secreted glycosyl transferases (GT), which participate in the formation of glycosidic bonds, were similarly low-abundant in the three isolates (Supplementary Table S3).

In addition to CAZymes, we identified candidate secreted effector proteins (CSEPs) and secreted proteases in the secretomes of the three fungal strains (Table 2). CSEP was the most abundant

Table 2. Functional classification of pathogenesis related genes in the three Plectosphaerella genomes studied

\begin{tabular}{lrrr}
\hline & \multicolumn{3}{c}{ Number of predicted } \\
& \multicolumn{3}{c}{ genes } \\
\cline { 2 - 4 } Annotated function & PcBMM & Pc2127 & P0831 \\
\hline Carbohydrate-active enzymes (CAZymes) & 782 & 782 & 768 \\
Secretome & 539 & 502 & 492 \\
$\quad$ Secreted CAZymes & 178 & 40 & 35 \\
Candidate secreted effector proteins & 253 & 221 & 217 \\
$\quad$ Proteases & 63 & 59 & 68 \\
Secondary metabolism & 87 & 79 & 82 \\
Transport & 1,032 & 1,015 & 1,114 \\
Total number of predicted genes in each & 11,323 & 11,007 & 10,821 \\
genome & & & \\
\hline
\end{tabular}

secreted category in the three genomes, representing between 44 and $47 \%$ of the predicted genes in the secretome (Table 2). No significant differences between the three isolates were found for CSEPs or proteases. We identified 87, 79, and 82 genes related to secondary metabolism pathways in the genomes of PcBMM, Pc2127, and P0831, respectively (Table 2; Supplementary Fig. S7). These pathways were represented by 15 core genes in each of the three genomes, which is half the average number of core genes found in other ascomycetes (Wang et al. 2015). P0831 contained more genes encoding nonribosomal peptide synthase (NRPS)-like genes and fewer genes encoding NRPSs and polyketide synthases than PcBMM and Pc2127 (Supplementary Fig. S6), but most of these genes had orthologous genes in the three genomes and only one gene was exclusive for each genome. These results suggest that the pathogenicity mechanisms of Plectosphaerella spp. in Arabidopsis (e.g., PcBMM strain) involve secreted CAZymes and probably proteinaceous toxins rather than toxins derived from secondary metabolites.

We also found 342, 264, and 387 genes, respectively, in PcBMM, Pc2127, and P0831 without homology to genes in other organisms. The proportion of these genes in the genome was significantly larger in P0831 $\left(P<0.00001, \chi^{2}\right)$. In PcBMM, one third of these genes (118) were isolate-exclusive, whereas only 35 and 17 genes were isolate-exclusive in Pc2127 and P0831, respectively.

\section{Differential repertoires of fungal genes are expressed during colonization of Col-0 and cyp79B2 cyp79B3 plants by Plectosphaerella strains.}

To identify Plectosphaerella genes determining the different types of interactions with Arabidopsis (Fig. 1), we performed genome-wide RNA-seq expression profiling. For that, we used total RNA extracted from Col-0 and cyp79B2 cyp79B3 threeweek-old plants at 10 and $16 \mathrm{~h}$ postinoculation (hpi) with spores $\left(4 \times 10^{6}\right.$ spores per milliliter) of PcBMM, Pc2127, or P0831 strains or after water treatment (noninoculated). In parallel, total RNA was also extracted from mycelia of the Plectosphaerella strains grown in vitro under minimal medium conditions. RNA-seq of in-vitro samples generated 59,794,266, $4,809,246$, and 7,343,450 mapped reads, which corresponded to 10,586, 10,282, and 10,272 expressed genes of the PcBMM, Pc2127, and P0831 strains, respectively (Supplementary Table S4). RNA-seq of in-planta samples generated from 36,324 to 189,858 fungal mapped reads ( 1 to $5 \%$ of the total sequenced reads), which reflects the relatively low fungal RNA abundance and the differences in growth ability of the strains in planta (Supplementary Table S4). In Col-0 inoculated plants, we detected the expression of 7,687, 7,188 and 3,236 genes of PcBMM, Pc2127, and P0831, respectively, which means 73, 70 , and $32 \%$ of the genes with detected expression (in vitro + in planta RNA-seq analyses [Fig. 3A and B]). These results showed dramatic differences for isolate P0831, which expressed in planta less than half of the number of genes expressed by PcBMM or Pc2127 (Fig. 3B).

We found specific clusters of genes from each fungal strain that were either up-regulated at different timepoints (10 or 16 hpi) or in one of the plant genotypes (Col-0 or cyp7B2 cyp79B3) (Fig. 3A). The number of PcBMM and Pc2127 genes expressed in cyp7B2 cyp79B3 plants increased at 16 hpi in relation to $10 \mathrm{hpi}$, whereas it decreased in Col-0 inoculated with Pc2127, as expected from the observed failure of this strain to grow in Col-0 plants (Figs. 1 and 3B). In contrast, we observed an increase in the number of fungal expressed genes in P0831 in Col-0 at 16 hpi in comparison with $10 \mathrm{hpi}$, further supporting the idea that the nature of the interaction of P0831 with Col-0 plants differs from that of Pc2127 (Fig. 1B). Notably, about $50 \%$ of the P0831 upregulated genes were expressed exclusively 
at 16 hpi (Supplementary Fig. S8A), while most of the upregulated genes of PcBMM and Pc2127 were expressed at both 10 and 16 hpi. Similarly, most PcBMM and Pc2127 upregulated genes were expressed in both Col-0 and cyp79B2 cyp79B3, whereas only $50 \%$ of the in planta-expressed P0831 genes were shared between both plant genotypes (Supplementary Fig. S8B). These data indicate that gene expression in P0831 was reprogrammed over time and depending on the immunity status of the host. To confirm RNA-seq data, in-planta expression of a representative subset of Plectosphaerella genes from some clusters were validated by quantitative reverse transcriptionPCR (qRT-PCR) in independent experiments at 10, 16, and 24 hpi (Fig. 3C).

We next analyzed the in planta expressed fungal genes that could be related to pathogenicity, and we found that the most represented category in the three strains was "membraneassociated transporters," followed by "secreted proteins," and "secondary metabolism pathways," which was in accordance with the relative abundance of these categories in the fungal genomes (Supplementary Fig. S9A; Table 2). The percentage of in planta-expressed genes within each category was higher in PcBMM and Pc2127 than in P0831 (28 to 36\% of PcBMM values [Supplementary Fig. S9A]), except for secondary metabolism pathways genes, whose expression was reduced about $60 \%$ in Pc2127 in comparison with PcBMM (Supplementary Fig. S9A). Main differences among strains were found within the number of genes of the secretome categories, particularly for the secreted CAZymes expressed in planta, which were significantly higher in PcBMM than in Pc2127 and P0831 (25 and 3\% of PcBMM values, respectively) (Supplementary Fig. S9B). Among the top 10\% of genes with the highest level of expression in planta, those encoding "secreted proteins," particularly secreted CAZymes, and "membrane associated transporters" were the most abundant in PcBMM and Pc2127, while in P0831 the most represented category of highest expressed genes was "membrane associated transporters," followed by "secreted proteins" (Supplementary Fig. S9C and D). These data support the relevant role of the secreted CAZymes in the pathogenicity of PcBMM and suggest that the survival strategy of P0831 on the plant surface could be based on the expression of membrane-associated transporters and on a weakened
A

PCBMM

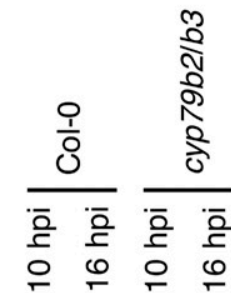

PC2127

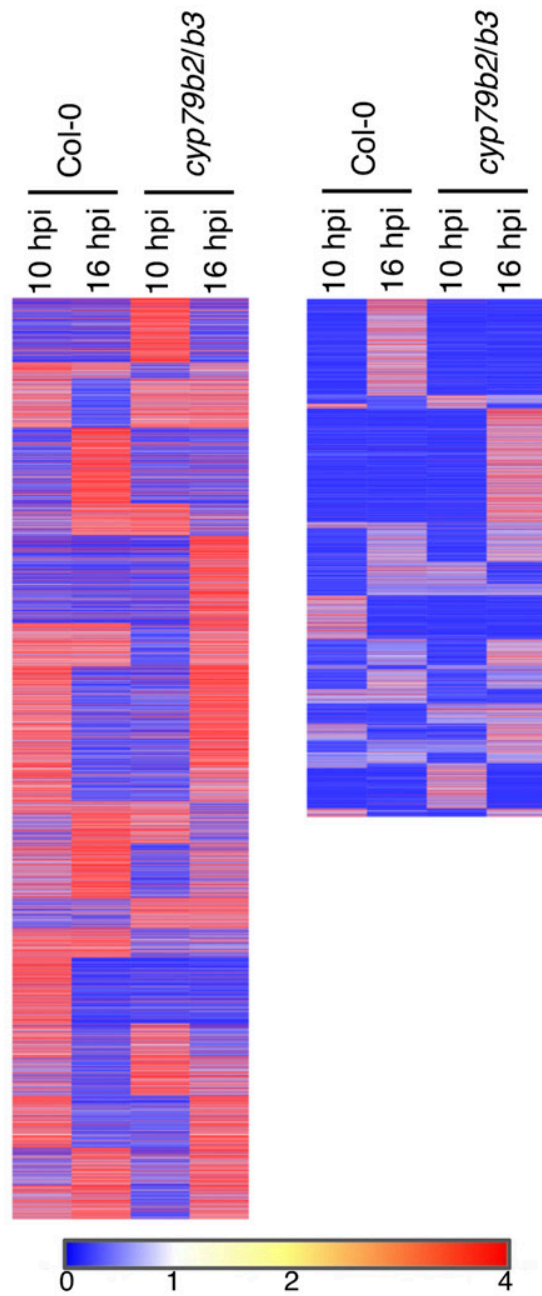

B

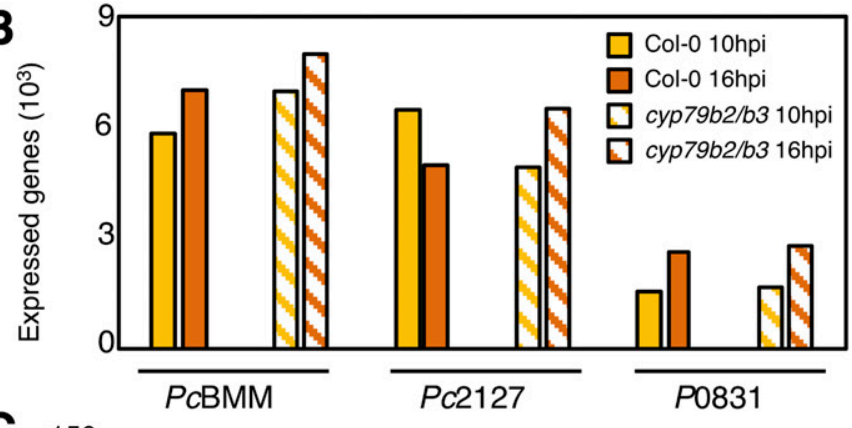

C
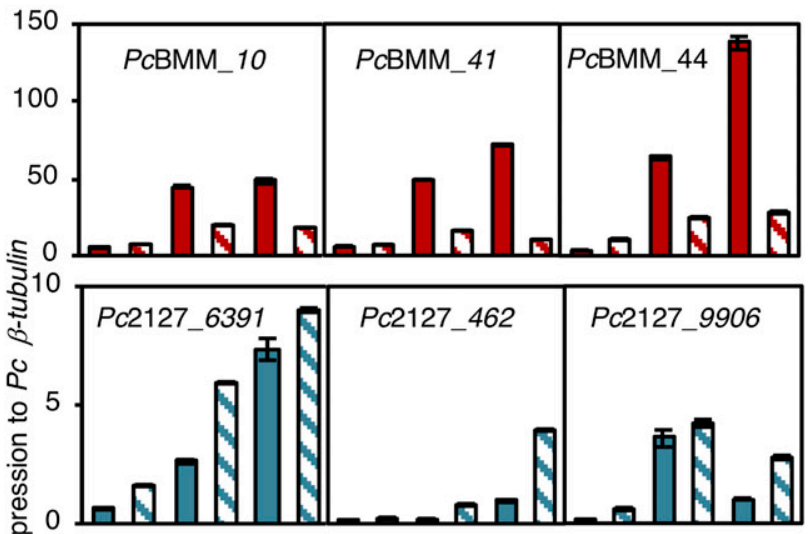

ำ

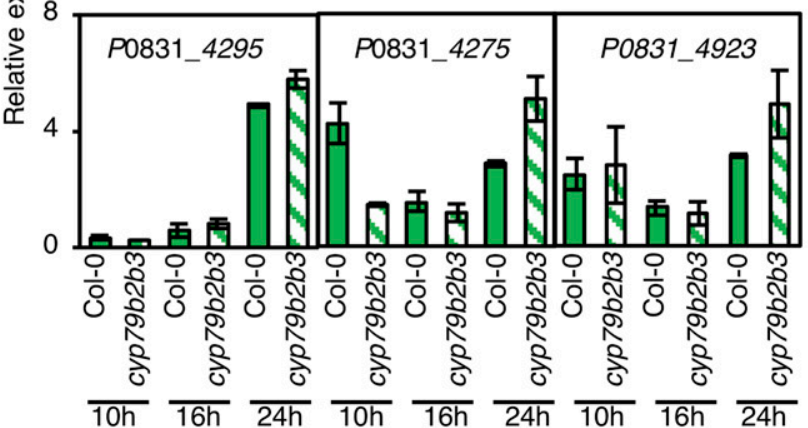

Fig. 3. Differential expression of the transcriptomes of PcBMM, Pc2127, and P0831 in Arabidopsis inoculated plants. A, Heatmaps of gene expression showing the fungal genes significantly upregulated ( $\log _{2}$-fold change $[\mathrm{FC}] \geq 2$, false discovery rate $\left.<0.05\right)$ in Col-0 and $c y p 79 B 2 \mathrm{cyp} 79 \mathrm{~B} 3$ inoculated plants at 10 and 16 $\mathrm{h}$ postinoculation (hpi) in relation to in-vitro growth. Overrepresented (white to dark red) and underrepresented transcripts (white to dark blue) are shown as $\log _{2}$-FC relative to the expression of the genes in vitro. B, Number of in planta-expressed genes of PcBMM, Pc2127, and P0831 in the tested conditions (Col0 and cyp79B2 cyp79B3) and timepoints (10 and $16 \mathrm{hpi}$ ). C, Validation by quantitative reverse transcription-PCR of the expression of some selected genes of PcBMM, Pc2127, and P0831 in Col-0 and cyp79B2 cyp79B3 plants at different timepoints (10 to 24 hpi). Values are the relative expression of selected genes to fungal $\beta$-tubulin gene expression levels. Bars represent the average ( \pm standard deviation) of two technical replicates. 
transcriptional activation of CAZyme genes. In general, minor differences in the expression of genes of the most represented pathogenicity categories were observed between Col-0 and cyp $79 b 2$ cyp79b3, with the exception of strain Pc2127, which expressed more secretome-associated genes when it infected the mutant (Supplementary Fig. S9B and C). This probably contributes to the colonization of this mutant by Pc2127 and further supports the relevance of these genes in fungal pathogenicity.

\section{Arabidopsis transcriptional responses during colonization by Plectosphaerella strains.}

Differential expression of Arabidopsis genes in Col-0 and cyp79B2 cyp79B3 plants upon inoculation (10 and $16 \mathrm{hpi}$ ) with the three fungal strains was determined by comparing RNA-seq data of these samples with those of noninoculated (mock) plants (Supplementary Tables S5 and S6). The total number of mapped RNA-seq reads and Arabidopsis-expressed genes detected in Col-0 and cyp79B2 cyp79B3 plants inoculated with each fungal strain was very similar, indicating that plant transcriptional responses were comparable (Fig. 4; Supplementary Table S5). We identified specific sets of differentially expressed genes (DEGs) (false discovery rate $[\mathrm{FDR}]<0.05$; $\log _{2}$-fold change $[\mathrm{FC}] \geq 1$ ) that were either upregulated or downregulated
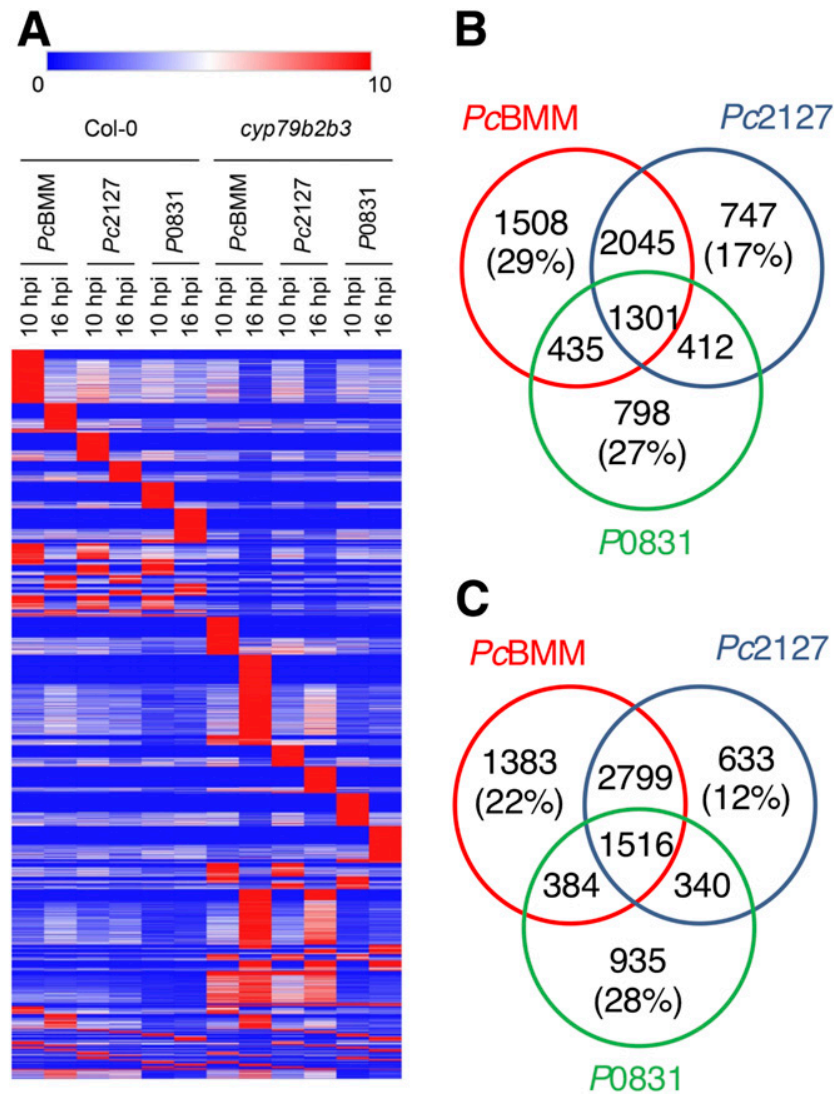

Fig. 4. Differential expression of the transcriptomes of Col-0 and cyp79B2 cyp $79 B 3$ plants inoculated with Plectosphaerella strains. A, Heatmaps of gene expression showing the plant genes significantly upregulated $\left(\log _{2}\right.$ fold change $[\mathrm{FC}] \geq 2$, false discovery rate $[\mathrm{FDR}]<0.05)$ in the Col-0 and cyp79B2 cyp79B3 inoculated plants at 10 and $16 \mathrm{~h}$ postinoculation (hpi). Overrepresented (white to dark red) and underrepresented transcripts (white to dark blue) are shown as $\log _{2}$-fold changes relative to the gene expression in mock-treated plants. $\mathbf{B}$ and $\mathbf{C}$, Venn diagrams showing the number of genes upregulated $\left(\log _{2}-\mathrm{FC} \geq 2\right.$, FDR $\left.<0.05\right)$ in Col-0 (B) or cyp79B2 cyp79B3 (C) plants at 10 and $16 \mathrm{~h}$ postinoculation with PcBMM, Pc2127, or P0831. The percentage of specific Arabidopsis genes upregulated by each fungal strain in inoculated Col-0 and cyp79B2 cyp79B3 plants is indicated between parentheses in the Venn diagram. in inoculated plants (Fig. 4A; Supplementary Table S6). Of note, the number of DEGs in P0831-inoculated plants was lower (from 35 to $48 \%$ ) than in plants infected with PcBMM or Pc2127 (Supplementary Table S6; Fig. 4B). The number of DEGs also differed among plant genotypes, since it was larger in cyp79B2 cyp79B3 than in Col-0 plants inoculated with any of the three strains (increases of 15,17 , and $7 \%$ for PcBMM Pc2127, and P0831, respectively) (Supplementary Table S6; Fig. 4B). Moreover, the number of plant upregulated DEGs increased over time in cyp $79 B 2$ cyp $79 B 3$ mutants inoculated with any of the strains and in Col-0 plants inoculated with PcBMM, whereas the number decreased in Col-0 plants inoculated with Pc2127 or P0831 (Supplementary Table S6; Supplementary Fig. S10). The number of Arabidopsis downregulated DEGs also increased over time in plants inoculated with PcBMM and Pc2127, especially in the cyp79B2 cyp79B3 mutant, whereas it remained stable in plants inoculated with P0831 (Supplementary Table S6). Interestingly, profound changes in the sets of DEGs (up- and downregulated) at 10 and 16 hpi were found, especially in plants inoculated with P0831, in which only 15 and $10 \%$ of DEGs were shared between the two timepoints tested in Col-0 and cyp79B2 cyp79B3 plants, respectively (Fig. 4A; Supplementary Fig. S10).

We identified two cores of 1,301 and 1,516 DEGs in Col-0 and cyp79B2 cyp79B3 plants, respectively, which were shared upon inoculation with the three fungal strains (Fig. 4A, B, and C; Supplementary Fig. S10). However, we also found specific clusters of DEGs upon infection progression, depending on the plant genotype and fungal strain, that represented from $29 \%$ of total DEGs in Col-0 inoculated with PcBMM to $12 \%$ in cyp79B2 cyp $79 B 3$ infected with Pc2127 (Fig. 4A, B, and C; Supplementary Fig. S10; Supplementary Table S6). The similarities between the transcriptional responses of plants inoculated with PcBMM and P0831 were lower (59\% of shared DEGs) than those between PcBMM and Pc2127 (over 74\% of shared genes [Fig. 4B and C; Supplementary Fig. S10]). Together, these data indicate that the transcriptional responses of Arabidopsis genotypes upon inoculation with each fungal strain differ significantly and that P0831 elicits in planta a weaker transcriptional response (lower number of DEGs) than the other two strains.

The putative functions of Arabidopsis DEGs in inoculated plants was analyzed by determining gene ontology (GO) term enrichment. DEGs in the Col-0-PcBMM interaction were enriched in $117 \mathrm{GO}$ terms that were mainly related to "defense," "signaling," and "response to stress" functions (Supplementary Table S7; Supplementary Fig. S11A). A very similar pattern was found for DEGs in Col-0-Pc2127 and cyp79B2 cyp79B3-PcBMM or cyp79B2 cyp79B3-Pc2127 interactions, although with fewer GO terms enriched (about 70 in the three cases [Supplementary Table S7; Supplementary Fig. S11A and B ]). Hence, a strong plant defense response is observed in these interactions independently of the final fungal outcome (e.g., disease progression or fungal growth inhibition). In cyp79B2 cyp79B3 plants, a remarkable enrichment in GO terms related to aging was also observed, which could reflect the activation of molecular mechanisms related to cell death associated to fungal-induced necrosis. A very different GO enrichment pattern was observed among the upregulated DEGs in the Col-0-P0831 interaction, with only eight GO terms, mainly related to metabolic processes such as "biosynthesis of secondary metabolites and toxins" that could be related to defense functions (Supplementary Table S7; Supplementary Fig. S11A). Similar GO term enrichment analyses were performed with downregulated DEGs, but the number of significant GO terms identified was very low, 14 and nine GO terms in the cyp79B2 cyp79B3 interactions with PcBMM and Pc2127, respectively, three GO termss in the Col-0-PcBMM interaction, 
and two in either Col-0 or cyp79B2 cyp79B3 P0831-inoculated plants (Supplementary Table S7).

The analyses of the number of DEGs and GO term enrichment indicated that the response of Col-0 plants to P0831 was significantly different from those to PcBMM and Pc2127 and, also, suggest that genes related to canonical immune and disease resistance response are either not induced or weakly expressed in plants inoculated with P0831. To further validate this hypothesis, we performed independent experiments in inoculated Col- 0 and cyp79B2 cyp79B3 plants to determine, by qRT-PCR, the expression of marker genes of different defense pathways (e.g., $P R I$ (SA), PDF1.2 (ET + JA), NCED3 (ABA), and PAD3, FRK1, and $C Y P 81 F 2$, which are PTI-associated genes). As shown in Supplementary Figure S12, the expression of the majority of these genes matched RNA-seq data and was strongly upregulated (up to 1,000-fold) in Col-0 and cyp79B2 cyp79B3 plants upon inoculation with PcBMM and Pc2127. In contrast, these defense and immunity marker genes were, in accordance with GO term enrichment analyses (Supplementary Fig. S1A), either not induced or were slightly up-regulated in P0831-inoculated plants (up to 10fold [Fig. S12]). These results further suggest that the immune responses of plants inoculated with the epiphytic strain are weakly induced or attenuated by the fungus.

\section{DISCUSSION}

Plant-fungus interactions are diverse and largely dependent on the genetic determinants of fungus and host and on the environmental conditions (Brader et al. 2017; Rai and Agarkar 2016; Zeilinger et al. 2016). Comparing closely related fungal species with different abilities to cause disease in different hosts can provide novel cues about the specific genetic determinants of pathogenesis (Hacquard et al. 2016; Seidl et al. 2015; Wang et al. 2020; Xu et al. 2014). In this work, we have compared the initial colonization steps of three strains of the genus Plectosphaerella on wild-type and immunocompromised Arabidopsis genotypes, and we have sequenced and annotated the genomes and studied the transcriptional responses of these fungi upon plant colonization. The comparative analysis of the pathogenic PcBMM strain and the two nonpathogenic strains P0831 and Pc2127 revealed that, beyond the expected remarkable differences between pathogenic and nonpathogenic interactions, nonpathogenic interactions are also highly distinct between them (Malcom et al. 2013; Selosse et al. 2018).

Among nonpathogenic interactions, we can distinguish endophytic and epiphytic fungal lifestyles (Arnold 2007; Hardoim et al. 2015; Yao et al. 2019). Fungal endophytes are a highly diverse group that naturally colonize internal plant tissues without causing disease symptoms for at least part of their life cycle (Hardoim et al. 2015) and have received considerable attention in recent years due to their potential benefits to plants (Brader et al. 2017; Fesel and Zuccharo 2016; Lugtenberg et al. 2016; Plett and Martin 2018). Endophytes share infection mechanisms with plant pathogens and can be potentially pathogenic in immunocompromised plants, such as the cyp79B2 cyp79B3 mutant (Hiruma et al. 2016). In contrast, the number of epiphytic fungal interactions characterized so far at the genomic and transcriptomic levels is scarce (Takashima et al. 2019; Wang et al. 2017; Xu et al. 2016). Fungal epiphytes are organisms growing on the leaf surface (Hongsanan et al. 2016), including pathogens and endophytes that can transiently colonize and be found on that habitat (Newton et al. 2010; Schulz and Boyle 2005). The leaf surface is a harsh environment, subject to abrupt temperature and humidity changes, scarcity of nutrients, and competitive forces in the diverse population of inhabiting microorganisms (Leveau 2015; Lindow and Brandl 2003). Host defenses are acting at the leaf surface, shaping the composition of epiphytic populations (Arnold 2007; Whipps et al. 2008). Therefore, epiphytes must have adaptations that allow their growth on the leaf surface and must interact with the host (Vorholt 2012; Wang et al. 2017; Xu et al. 2016). Here, we have sequenced, annotated, and characterized the transcriptomic response of the epiphytic strain P0831 in its interaction with Arabidopsis wild type (Col-0) and a cyp79B2 cyp79B3 mutant. Notably, we have found that strain P0831 is able to colonize Col-0 wild-type plants and also the immune-defective dde2 ein2 pad4 sid2 and cyp79B2 cyp79B3 mutants, without causing disease (Fig. 1). This contrasts with the observed phenotypes of PcBMM, which was more virulent in these immunocompromised mutants than in Col-0 plants, and of Pc2127, which, as previously described, was pathogenic on cyp79B2 cyp79B3 plants but not on Col-0 (Sánchez-Vallet et al. 2010). Thus, our data reveal a novel lifestyle (epiphytic) of Plectosphaerella spp. on Arabidopsis plants that had not been previously studied. Since P0831 was isolated from a natural population of Arabidopsis (García et al. 2013) and fungi of genus Plectosphaerella are consistently associated with Arabidopsis in nature (Thiergart et al. 2020), we can conclude that the P0831-Arabidopsis interaction described here occurs in nature.

Several studies on the interaction of $P$. cucumerina with Arabidopsis have largely contributed to a precise understanding of plant basal immunity against fungal necrotrophs (Bednarek et al. 2009; Hernández-Blanco et al. 2007; Lipka et al. 2005; Pastorczyk et al. 2020; Sánchez-Vallet et al. 2010; Stein et al. 2006). These studies found dramatic differences between strains in their ability to overcome plant defenses and to cause disease, but the genetic determinants of pathogenesis of these fungi had not been characterized so far. The genomic and transcriptomic analyses of the different strains of genus Plectosphaerella described here place this genus very close to Verticillium and Collectotrichum species and not phylogenetically related to other fungal genera like Botrytis or Sclerotinia that contain strains with necrotrophic lifestyles in different plant species, including Arabidopsis (Dickman and Mitra 1992; Ge and Barbetti 2019; Windram et al. 2012). We have found that the pathogenicity determinants in genus Plectosphaerella include mainly CWDE (including secreted CAZYmes) and proteinaceus toxins and effectors rather than secondary metabolites (Table 1; Supplementary Fig. S9), which contrasts with Botrytis or Sclerotinia species that used these metabolites as pathogenicity determinants (Amselem et al. 2011). These results suggest that the necrotrophic lifestyle of plant pathogenic fungi has been evolutionarily achieved through different genetic mechanisms. Our results also indicate that genetic differences among strains are more related to their taxonomic position than with their pathogenic ability, since we identified very few exclusive genes in each Plectosphaerella genome.

We have found a remarkable relationship between the number of secreted CAZymes in the Plectosphaerella strains and their pathogenicity on wild-type plants (Table 2; Supplementary Table S3; Supplementary Fig. S9), pointing to this group of enzymes as one of the main determinants of pathogenicity in Plectosphaerella spp. By contrast, the rest of the well-described pathogenicity determinants seem to be very well-conserved in the three fungal strains and similarly expressed in colonized plants (Table 2). CAZymes, which comprise several classes of proteins with different catalytic activities on carbohydrates (Lombard et al. 2014), are often classified as CWDEs, since they process or hydrolyze plant polysaccharides to either facilitate infection, gain access to nutrients, or both (Kameshwar et al. 2019; Zhao et al. 2013). For example, pathogens of dicots often contain more pectinases than fungi-infecting monocots, as dicots contain more pectins in their cell walls than monocots (Zhao et al. 2013). CAZymes have been previously identified as important pathogenicity or virulence factors of necrotrophic fungi (Chang et al. 2016; Douaiher et al. 2007; Kikot, et al. 
2009; Lyu et al. 2015). Biotrophic fungi tend to have fewer CAZymes than necrotrophic and hemibiotrophic fungi, which is consistent with their lifestyles (Lyu et al. 2015; Zhao et al. 2013). While nutrient acquisition of necrotrophic and hemibiotrophic fungi is based on killing the host cell at the last stage of infection, biotrophic fungi absorb nutrients from living cells (Laluk and Mengiste 2010; Oliver and Ipcho 2004). The genomes of the three Plectosphaerella strains studied here harbor similar numbers of CAZymes (Table 2), also in line with those found in other necrotrophic and hemibiotrophic phytopathogenic fungi of dicots (Gazis et al. 2016; Knapp et al. 2018; Wang et al. 2020; Xu et al. 2014). However, the numbers of GT, $\mathrm{CE}, \mathrm{GH}$, and PL CAZymes in Plectosphaerella genomes are higher than in other phytopathogenic fungi, except for some Colletotrichum and Fusarium species (Hacquard et al. 2016). For example, CEs are up to three times more abundant in Plectospaherella genomes than in other phytopathogenic fungi (Table 2). CEs catalyze the de- $O$ or de- $N$-acylation of esters or amides and other substituted saccharides in which sugars play the role of alcohol and amine (Biely 2012). Acetylation of glycosyl residues of polysaccharides prevents hydrolysis of their glycosidic linkages by the corresponding $\mathrm{GH}$, protecting plant cell walls against invading microorganisms. For instance, Arabidopsis eskl or rwa/pmr mutants show alterations in enzymes related to plant cell-wall polysaccharide acetylation and differential disease resistance responses to pathogens, including PcBMM (Escudero et al. 2017; Pawar et al. 2016; Yuan et al. 2013). Conversely, acetyl groups became targets of microbial CE that evolved to overcome the complexity of the plant cell walls and that cooperate with $\mathrm{GH}$ in plant polysaccharide degradation. Of note, plants overexpressing fungal esterases and acetylases show differential immune responses and disease-resistance phenotypes (Pawar et al. 2016).

In addition to the catalytic modules, around $7 \%$ of the CAZymes of genus Plectosphaerella also contain CBMs, which are the most common noncatalytic modules associated with enzymes active in cell-wall hydrolysis (Lombard et al. 2014). Among these CBMs are LysM-bearing proteins (CBM50 family) that have been described to function in scavenging chitin and other $\beta$-glucan fungal oligomers that are perceived as MAMPs by the plant immune system, thus preventing the recognition of the fungus by plant PRRs (Bolton et al. 2008; de Jonge and Thomma 2009). The number of CBM domains found in the three Plectosphaerella strains were not different, but the number of secreted proteins with these modules were seven and 18 times higher in PcBMM than in Pc2127 and P0831, respectively (Supplementary Table S3), probably explaining the ability of PcBMM to overcome plant immune responses.

Fungal secretomes play crucial roles during plant colonization by necrotrophic, hemibiotrophic, or biotrophic fungi and oomycetes (Kamoun 2009; Kim et al. 2016; Lyu et al. 2015; Stergiopoulos and de Wit 2009). The function of secreted proteins in necrotrophic fungi with a broad host range is poorly understood (Guyon et al. 2014). Secretome analysis of the necrotrophic fungus Sclerotinia sclerotiorum revealed large numbers of predicted effector candidates in the genome and the crucial role of nonCWDE secreted proteins during infection (Lyu et al. 2016). Here, we show that there are no significant differences between the number of small secreted proteins encoded by the genomes of the three Plectosphaerella strains. This contrasts with the differences observed in the number of genes encoding predicted secreted CAZymes (Table 2), which is much higher in PcBMM (Table 2). Moreover, these secreted CAZymes are expressed during the colonization process in higher numbers in pathogenic PcBMM than in the nonpathogenic strains (Supplementary Fig. S9). Therefore, we can conclude that secreted CAZymes are determinants of PcBMM pathogenicity.
RNA-seq analysis showed dramatic differences in fungal and plant gene expression at early stages of the interactions studied that not fully correlated with the ability of the different strains to grow and cause disease in the plant. The P0831 strain expressed in planta half of the genes expressed by PcBMM or Pc2127 (Fig. 3). The number of in planta-expressed genes of PcBMM or P0831 was in line with results obtained in inoculated plants with pathogenic and nonpathogenic strains of other fungi, such as Colletotrichum or Fusarium species (Baetsen-Young et al. 2020; Hacquard et al. 2016). However, in the case of Pc2127, the number of expressed genes was closer to the pathogenic interaction caused by PcBMM, suggesting an aggressive behavior of this strain at short timepoints. Pc2127, however, does not thrive at later timepoints (Fig. 3), and the number of its expressed genes decreased at $16 \mathrm{hpi}$ in Col-0 inoculated plants, which contrasted with the stable or increased number of expressed genes of PcBMM or P0831 over time. These profound differences in gene expression dynamics between Pc2127 and P0831 strains in Col-0 Arabidopsis indicate the different nature of their interactions with Arabidopsis besides being both nonpathogenic.

Pc2127 was highly virulent on immunocompromised cyp79B2 cyp $79 B 3$ plants but did not cause disease on dde 2 ein 2 pad4 sid2 mutants (Fig. 1), indicating that Trp-derived metabolites rather than ET, SA, and JA and ETI pathways are required for basal resistance to nonadapted Plectosphaerella fungi, as previously described (Bednarek et al. 2009; SánchezVallet et al. 2010). P0831 was able to grow on plant surfaces both of wild type and immunocompromised Arabidopsis mutants without causing disease symptoms (Fig. 1; Supplementary Figs. S2 and S3). These results suggest that alternative plant defense mechanisms might prevent plant infection by P0831. However, our results also showed a weaker upregulation of canonical plant immune responses upon P0831 epiphytic growth when compared with the other two strains (Supplementary Figs. S11 and S12). This weak immune response does not seem to be related to a defective perception of P0831, since in-vivo analyses at 3 dpi with $p R b o h D:: L U C$ plants demonstrate that these plants are able to perceive this fungus and to activate transcriptional responses (Supplementary Fig. S4). These results suggest that the epiphytic P0831 strain might inhibit activation of the plant immune system at early stages of colonization through specific mechanisms that will require further characterization. The transcriptional response of P0831 upon interaction with Arabidopsis was distinct from those of PcBMM and Pc2127, and it changed considerably over time and depending on the immunity status of the host (Fig. 3; Supplementary Fig. S8). Strikingly, the transcriptional response of P0831 seemed more related to transport than with pathogenicity (Supplementary Fig. S9). In summary, we have shown here the diversity of interactions that Plectosphaerella fungal strains can establish with plants (Arabidopsis) and have characterized a novel type of epiphytic interaction. The characterization of Plectosphaerella genomes and transcriptome has also revealed that the expression of specific sets of fungal genes in planta determines the diverse lifestyles and pathogenicity of the Plectosphaerella strains studied.

\section{MATERIALS AND METHODS}

\section{Detection of Plectosphaerella sp. from natural A. thaliana populations.}

Asymptomatic A. thaliana leaves and roots were collected from natural populations in central Spain, Menasalbas (MEN) and Las Rozas, that have been described previously (García et al. 2013). Total DNA was extracted from the samples using the FastDNA SPIN Kit for Soil (MP Biomedicals) and $10 \mathrm{ng}$ of 
DNA template was used for real time PCR amplification using the specific primer pair that targets the Plectosphaerella $\beta$ tubulin gene, 5' -CAAGTATGTTCCCCGAGCCGT-3' and 5' GGTCCCTTCGGTCAGCTCTTC-3' (Sánchez-Rodríguez et al. 2009), and the FS Universal SYBR Green Master Rox (Roche) as described (Delgado-Cerezo et al. 2012).

\section{Biological material and growth conditions.}

A. thaliana genotypes used in this study were accession Columbia-0 (Col-0) and the mutants (in Col-0 background) cyp79B2 cyp79B3 (Zhao et al. 2002), dde2 ein2 pad4 sid2 (Tsuda et al. 2009), and the pRBOHD::LUC line (in Col-0 background [Morales et al. 2016]). Plants were grown on a 3:1 soil-vermiculite mixture under a 10 -h day and 14-h night cycle, $24^{\circ} \mathrm{C}$ day and $22^{\circ} \mathrm{C}$ night temperatures, $65 \%$ relative humidity, and light intensity of $120 \mathrm{mE} \mathrm{m}^{-2} \mathrm{~s}^{-1}$. PcBMM isolate was provided by B. Mauch-Mani (University of Neuchâel, Switzerland [Tierens et al. 2001, Ton and Mauch-Mani 2004]), and Pc2127 was obtained from the Deutsche Sammlung von Mikroorganismen und Zelkulturen $\mathrm{GmbH}$ (DSMZ) Collection (Braunschweig, Germany). P0831 strain was isolated from an asymptomatic Arabidopsis leaf from the wild MEN population in central Spain (García et al. 2013). Spores from these fungi were obtained as reported by Ramos et al. (2013). P0831-GFP transformant was obtained and selected following previously reported methodology used to obtain PcBMM-GFP and Pc2127-GFP transformants (Ramos et al. 2013).

\section{Arabidopsis inoculation and detection of Plectosphaerella isolates.}

Inoculation of Arabidopsis leaves was performed by spraying 3week-old soil-grown plants with a spore suspension $\left(4 \times 10^{6}\right.$ spores per milliliter) of the fungus as described (Llorente et al. 2005). At least 12 plants per genotype were inoculated and a minimum of three independent experiments were performed. For fungal DNA quantification, DNA from infected plants was extracted as described by Llorente et al. (2005) and fungal DNA was determined by qPCR using the FS Universal SYBR Green Master Rox (Roche), oligonucleotides for the Plectosphaerella $\beta$-tubulin gene, and the Arabidopsis UBIQUITIN21 (At5G25760, UBQ21) gene, and by calculating the change in the cycle threshold $(\Delta \mathrm{Ct})$ values, as reported previously (Delgado-Cerezo et al. 2012). The relative ratio was determined from the expression $2^{-\Delta \Delta C t}$ (Rieu and Powers 2009). The qPCR results are the means ( \pm standard deviation $[\mathrm{SD}]$ ) of two technical replicates.

Confocal images were acquired on a TCS SP2 AOBS spectral confocal laser scanning microscope (Leica Microsystems) as described (Ramos et al. 2013). GFP fluorescence (green) was captured by excitation at $488 \mathrm{~nm}$ and emission in the range 490 to $520 \mathrm{~nm}$, and chlorophyll autofluorescence (red) was captured with an excitation of $543 \mathrm{~nm}$ and the emission was collected in the range 600 to $720 \mathrm{~nm}$. The dimensional $x y z$ confocal stacks and orthogonal projections across selected $x z$ and $y z$ planes were obtained using Leica Confocal Software LCS Lite version 2.61 build 1538 (Leica Microsystems).

Lactophenol-TB staining of plant leaves was performed as described by Koch and Slusarenko (1990) and detection of $\mathrm{H}_{2} \mathrm{O}_{2}$ by 3.3'-DAB (Sigma) staining was performed as described by Torres et al. (2002). For in vivo quantification of Rboh-promoter driven LUC activity, pRBOHD::LUC Col-0 plants were sprayed with a $0.5 \mathrm{mM}$ solution of D-luciferin (Melford) and luminescence produced was measured using a NightOWL LB 983 in-vivo imaging system (Berthold) as described by Morales et al. (2016).

\section{Genome sequencing and assembly of Plectosphaerella strains.}

Library construction, quality control, and DNA Illumina Hiseq sequencing (PE $125 \mathrm{bp}$ and SE $50 \mathrm{bp}$ ) were performed at the
Centre for Genomic Regulation (CRG) (Barcelona, Spain) in an Illumina HiSeq2500, and PacBio sequencing was performed at Wageningen University and Research (Wageningen, The Netherlands), using $1 \mu \mathrm{g}$ of fungal genomic DNA. For fungal DNA extraction, cultures were grown at $28^{\circ} \mathrm{C}$ as described previously (Ramos et al. 2007). For PcBMM genome assembly, a hybrid strategy was used combining Illumina and PacBio data. SE and PacBio reads were first assembled using Velvet 1.2.10 (Zerbino and Birney 2008) and were further used for scaffolding of Illumina PE read assemblies from SPAdes assembler version 3.11.0 (Bankevich et al. 2012). The established SPAdes pipeline was used in careful mode providing Illumina and PacBio assemblies as untrusted contigs for scaffolding only and kmer scan using 21, 31, 41, 51, 61, 71, and 81. For Pc2127 and P0831, assemblies were constructed only from Illumina data using a combination of Velvet 1.2.10 (Zerbino and Birney 2008) and SPAdes version 3.11.0 (Bankevich et al. 2012). To identify and remove potential contaminating sequences, assemblies were aligned to the genomes of A. thaliana and Homo sapiens using MUMmer v. 3.0 (Kurtz et al. 2004) with default parameters settings. Contigs that aligned with more than $50 \%$ of their sequence or at least $85 \%$ sequence identity to any of the selected genomes were removed from the assemblies. Finally, assembly quality was assessed on the basis of L50/75 and $\mathrm{N} 50 / 75$ values, percentage of error-free bases estimated with quast 5.0.1 (Gurevich et al. 2013), and gene space coverage estimated with BUSCO v3 (Simão et al. 2015). The Whole-Genome Shotgun projects have been deposited in the DDBJ/ENA/GenBank database under accessions JACAFV000000000 (Plectosphaerella sp. strain P0831 Biosample SAMN14235337 TaxID: 40657), JACAFW000000000 (P. cucumerina Pc2127 Biosample SAMN14233823 and Pc2127 TaxID: 40658), SAMN14233823 and Pc2127 TaxID: 40658), and JACAFX000000000 (P. сисиmerina PcBMM Biosample SAMN14233534 TaxID: 40658), and BioProject number PRJNA609142. The versions described by this paper are versions JACAFV010000000, JACAFW010000000, and JACAFX010000000.

\section{Gene annotation of Plectosphaerella strains.}

The prediction of PcBMM, Pc2127, and P0831 gene models was performed using the Maker v2.31.10 pipeline (Cantarel et al. 2008) that integrates different $a b$ initio gene prediction tools together with evidence from expressed sequence tag and protein alignments. In a first step for each genome, the pipeline was run using Augustus (Stanke et al. 2006) (with species model Fusarium graminearum) and GeneMark-ES (Hoff et al. 2016) for $a b$ initio gene prediction together with transcript and protein alignment evidence. The resulting gene models from this first run were used as training set for a third $a b$ initio prediction tool, SNAP (Korf 2004), and, subsequently, the annotation pipeline was rerun, this time including all three $a b$ initio prediction tools together with the transcript and protein alignment evidence to yield the final gene models. The alignment evidence was created from BLAST and Exonerate (Zaharia et al. 2011) alignments of both protein and transcript sequences of each respective fungus obtained from corresponding RNA-seq data via a transcriptome de novo assembly. For this purpose, we extracted RNA-seq reads that did not align to the host plant genome from in-planta samples and combined these with the reads from in-vitro samples of the respective fungus. The combined RNA-seq reads were used as input for Trinity (Bryant et al. 2017), with default parameter, to assemble transcripts and extract peptide sequences of the best-scoring open reading frames. General functional annotations for the predicted gene models were obtained using Blast2GO (Conesa et al. 2005) with the local National Center for Biotechnology Information (NCBI) Nonredundant database downloaded (version: June 12, 2018).

\section{Read mapping and variant calling.}

Read mapping and variant calling was performed using the PcBMM assembly as reference. Before aligning the reads 
against the reference assembly, they were trimmed at the first base with a PHRED quality below 10 and pairs with one or both reads $<31$ bases after trimming were filtered out. Then, Pc2127 and P0831 reads were aligned against PcBMM assembly using BWA-MEM algorithm of BWA v0.7.13 (Li 2013) and were sorted with SAMtools v0.1.18 (Li et al. 2009). GATK v3.5 (McKenna et al. 2010) was used to call SNPs, and they were filtered according to GATK documentation $(\mathrm{QD}<2.0$; MQ $<$ 40; FS > 60.0; haplotype score $>13.0$; MQRankSum $<-12.5$; ReadPosRankSum <-8.0).

To detect putative deletions, a mpileup file of each strain (Pc2127 and P0831) was generated with SAMtools v0.1.18 (Li et al. 2009) and was compared with the reference gff file. A base was considered to be present in the strain if it was covered by at least one read. The percentage of total coverage for a region was calculated through the ratio between the number of positions with reads and the total size of the respective region. To obtain the identity between each strain and the reference genome, a BLASTp (Zhang et al. 2000) between the strain proteome and the reference and vice versa was performed. Only results in which both BLASTp best hits were the same were considered for further analysis. Then, the identity of each strain was determined with trimAl v1.4.rev15 (Capella-Gutierrez et al. 2009).

\section{Phylogeny of different species in the genus Plectosphaerella.}

Phylogeny was obtained with the maximum likelihood method and Tamura-Nei model (Tamura and Nei 1993) on a multilocus alignment (TUB, EF1, and ITS). One or more initial trees for the heuristic search were obtained automatically by applying neighbor-joining and BioNJ algorithms to a matrix of pairwise distances estimated using the maximum composite likelihood (MCL) approach and then selecting the topology with superior log likelihood value. The tree was drawn to scale, with branch lengths measured in the number of substitutions per site. This analysis involved 28 nucleotide sequences and there were a total of 1,004 positions in the final dataset. Evolutionary analyses were conducted in MEGA X (Kumar et al. 2018).

\section{MCL analysis and whole-genome phylogeny.}

Gene families and clusters of orthologous genes were inferred, using OrthoFinder (Emms and Kelly 2015) v2.2.7, with standard parameters, using Diamond (Buchfink et al. 2015) as a sequence aligner for protein and translated DNA searches. Whole-genome phylogeny was inferred by using the STAG method (Emms and Kelly 2018) with Orthofinder algorithm within each orthogroup of genes present in all the 27 compared genomes (Supplementary Table S1). A greedy consensus tree was achieved from all these individual estimates to get the final tree.

\section{Phylome reconstruction and gene gain and loss analysis.}

The Plectosphaerella phylome was reconstructed with PhylomeDB (Huerta-Cepas et al. 2014). For each Plectosphaerella protein, a Smith-Waterman search was performed against the proteome database containing data on the 24 species used in this work (Supplementary Table S1), as well as of the three Plectosphaerella strains. To obtain a set of proteins with significant similarity, an e-value threshold of $<1 \mathrm{e}-05$ was established. Moreover, only sequences that aligned with a continuous region longer than $50 \%$ of the query sequence were chosen for the detection of homologs. At maximum, 150 sequences per gene were taken for further analysis. MUSCLE v3.8.31 (Edgar 2004), MAFFT v6.814b (Katoh et al. 2002), and DIALIGN-TX (Subramanian et al. 2008) were used to align, in forward and reverse directions, the homologous protein sequences. M-COFFEE (T-Coffee v8.80) (Wallace et al. 2006) was used to combine the six resulting alignments. The final alignment was trimmed with trimAl v1.3 (consistency cutoff of 0.1667 and a gap score cutoff of 0.1) (Capella-Gutierrez et al. 2009). Phylogenetic trees were reconstructed through a neighbor-joining approach as implemented in BioNJ (Gascuel 1997). Eight different models (JTT, WAG, MtREV, VT, LG, Blosum62, CpREV, and DCMut) were used to compute the likelihood of this topology, allowing branch-length optimization, as in PhyML v3.0 (Guindon et al. 2010). Their likelihood was compared in accordance with the AIC criterion, and the two evolutionary models best fitting the data were selected to derive maximum likelihood trees. A discrete gamma-distribution model with four rate categories plus invariant positions was used in all cases, with parameters estimated from the data. Gene gain and loss analysis in the Plectosphaerella branch was performed based on the phylome results. For each gene from the starting genome, orthologs were mapped using a species overlap algorithm. Then the emergence of each gene was located at the common ancestor of all its orthologs. Losses were inferred by going from the node where the gene was gained to the tips and locating nodes where all species had lost the gene. GO term enrichment was calculated using an inhouse adaptation of FatiGo (Al-Shahrour et al. 2004). Enrichment was searched between genes gained and lost in the nodes pertaining to the emergence and diversification of Plectosphaerella species.

\section{Annotation of specific gene categories.}

To predict the repertoire of CAZYmes encoded by the three strains, the corresponding genomes were scanned using the CAZYmes analysis toolkit (Park et al. 2010). The secretomes were determined using the SECRETOOL pipeline (Cortázar et al. 2014) run on the proteome of each strain. CSEPs were defined as extracellular secreted proteins with no significant BLAST similarity (e-value $<1 \mathrm{e}-03$ ). To identify genes encoding secreted proteases, sequences of predicted extracellular secreted proteins were subjected to MEROPS Batch BLAST analysis (Rawlings et al. 2016). Putative membrane transporter genes were identified and classified through BLAST searches against the Transporter Collection (TC) database (Saier et al. 2014). Genes with a sequence identity of at least $30 \%$ to their best hit in the database (e-value $<1 \mathrm{e}-03$ ) were extracted. These genes were subsequently assigned to the TC family of their best TC database hit. Secondary metabolism was predicted using the antiSMASH fungal version web server (Medema et al. 2011).

\section{RNA-seq and gene expression analyses.}

Library construction, quality control, and RNA Illumina Hiseq 50-bp SE sequencing were performed at the CRG in an Illumina HiSeq2500 using $1 \mu \mathrm{g}$ of total RNA. To make sure the sequenced reads were of sufficiently high quality, an initial quality check was performed using the FastQC suite (Andrews 2010). All statistical analyses of plant and fungal gene expression were performed using Tophat and the Cufflinks pipeline (Trapnell et al. 2012), where the RNA-seq reads were mapped to the assembled and annotated genomes of either PcBMM, Pc2127, or P0831 and in parallel to the annotated genome of the host plant $A$. thaliana (ARAPORT). Differential gene expression in Col-0 and cyp79B2 cyp79B3 plants inoculated with PcBMM, Pc2127, and P0831 was fitted for each analysis using FPKM (fragments per kilobase per million reads) values. Heatmaps of gene expression profiles were generated with the Morpheus analysis package. Gene expression data of fungal and plant genes are shown in Supplementary Tables S8 and S9. The raw RNA-seq data in this study are deposited in the NCBI Sequence Read Archive (SRA), BioProject number PRJNA614936 and BioSamples SAMN14444085 to SAMN14444096, SAMN14444149, SAMN14444167, and SAMN14444168, with SRA accessions SRR11668188 to SRR11668202. 
The cytoscape plugin Cluego + Cluepedia (Bindea et al. 2009) was used to construct GO term enrichment networks and to visualize functionally grouped terms among significantly regulated genes (FDR $<0.05 ; \log _{2}-\mathrm{FC} \geq 1$ ) in Arabidopsis inoculated plants compared with mock-inoculated plants. Significant $(P<0.05)$ enrichments were determined using the hypergeometric test and were manually classified as defense, signaling, response to stress, metabolic process, transcription and translation, multiorganism process, or aging (Supplementary Table S7).

\section{Gene expression validation.}

RNA extractions from Plectosphaerella- or mock-inoculated plants were done as described by Llorente et al. (2005). Realtime qRT-PCR analyses were done as described previously (Delgado-Cerezo et al. 2012), using the FS Universal SYBR Green Master Rox (Roche). The qRT-PCR results are the means $( \pm \mathrm{SD})$ of two technical replicates. Oligonucleotides used for cDNA amplification were designed with Primer Express (version 2.0; Applied Biosystems) and are shown in Supplementary Table S10 (Jordá et al. 2016; Sánchez-Rodríguez et al. 2009). For fungal genes, relative expression was calculated related to the $\beta$-tubulin expression. For Arabidopsis genes, ubiquitin (AT5G25760) expression was used to normalize the transcript level in each sample of the genes analyzed. The relative ratio was then determined from the expression $2^{-\Delta \Delta \mathrm{Ct}}$ (Rieu and Powers 2009), using the mock-inoculated plants as calibrators.

\section{ACKNOWLEDGMENTS}

We thank L. Faino and B. Thomma (Laboratory of Phytopathology, Wageningen University \& Research, The Netherlands) for providing support with Plectosphaerella paired-ends sequencing, and A. Vlasova, F. Cámara and R. Guigó (CRG, Barcelona Institute of Science and Technology, Barcelona, Spain) for helping with the initial draft genome assemblies. We thank P. del Prado (CBGP, UPM-INIA) for technical assistance.

\section{AUTHOR-RECOMMENDED INTERNET RESOURCES}

antiSMASH fungal version web server:

https://fungismash.secondarymetabolites.org/\#!/start

ARAPORT Arabidopsis Information Portal: https://www.araport.org

Augustus gene prediction program: http://bioinf.uni-greifswald.de/augustus

Blast2GO: https://www.blast2go.com

BUSCO v3: https://busco.ezlab.org

Cytoscape Cluego: http://apps.cytoscape.org/apps/cluego

Diamond: https://github.com/bbuchfink/diamond

Exonerate software: https://www.ebi.ac.uk/about/vertebrate-genomics/ software/exonerate

FastQC suite: https://www.bioinformatics.babraham.ac.uk/projects/fastqc GeneMark-ES: http://exon.gatech.edu/GeneMark

MEROPS database: https://www.ebi.ac.uk/merops/submit_searches.shtml Morpheus software: https://software.broadinstitute.org/morpheus

MUMmer v. 3.0: http://mummer.sourceforge.net

OrthoFinder: http://www.stevekellylab.com/software/orthofinder

SNAP prediction tool: http://snap.cs.berkeley.edu

SECRETOOL pipeline:

http://genomics.cicbiogune.es/SECRETOOL/Secretool.php

Cufflinks pipeline: http://cole-trapnell-lab.github.io/cufflinks/

Transporter Collection database: http://www.tcdb.org

Trinity de novo assembly: https://github.com/trinityrnaseq/trinityrnaseq/wiki

\section{LITERATURE CITED}

Al-Shahrour, F., Díaz-Uriarte, R., and Dopazo, J. 2004. FatiGO: A web tool for finding significant associations of gene ontology terms with groups of genes. Bioinformatics 20:578-580.

Amselem, J., Cuomo, C. A., van Kan, J. A., Viaud, M., Benito, E. P., Couloux, A., Coutinho, P. M., de Vries, R. P., Dyer, P. S., Fillinger, S., Fournier, E., Gout, L., Hahn, M., Kohn, L., Lapalu, N., Plummer, K. M., Pradier, J. M., Quévillon, E., Sharon, A., Simon, A., ten Have, A., Tudzynski, B., Tudzynski, P., Wincker, P., Andrew, M., Anthouard, V.,
Beever, R. E., Beffa, R., Benoit, I., Bouzid, O., Brault, B., Chen, Z., Choquer, M., Collémare, J., Cotton, P., Danchin, E. G., Da Silva, C., Gautier, A., Giraud, C., Giraud, T., Gonzalez, C., Grossetete, S., Güldener, U., Henrissat, B., Howlett, B. J., Kodira, C., Kretschmer, M., Lappartient, A., Leroch, M., Levis, C., Mauceli, E., Neuvéglise, C., Oeser, B., Pearson, M., Poulain, J., Poussereau, N., Quesneville, H., Rascle, C., Schumacher, J., Ségurens, B., Sexton, A., Silva, E., Sirven, C., Soanes, D. M., Talbot, N. J., Templeton, M., Yandava, C., Yarden, O., Zeng, Q., Rollins, J. A., Lebrun, M. H., and Dickman, M. 2011. Genomic analysis of the necrotrophic fungal pathogens Sclerotinia sclerotiorum and Botrytis cinerea. PLoS Genet. 7:e1002230.

Andrews, S. 2010. FastQC: A quality control tool for high throughput sequence data. Babraham Bioinformatics, Babraham Institute, Cambridge. http://www. bioinformatics.babraham.ac.uk/projects/fastqc.

Arnold, A. E. 2007. Understanding the diversity of foliar endophytic fungi: Progress, challenges, and frontiers. Fungal Biol. Rev. 21:51-66.

Bacete, L., Mélida, H., López, G., Dabos, P., Tremousaygue, D., Denancé N., Miedes, E., Bulone, V., Goffner, D., and Molina, A. 2020. Arabidopsis response regulator 6 (ARR6) modulates plant cell wall composition and disease resistance. Mol. Plant-Microbe Interact. 33: 767-780.

Bacete, L., Mélida, H., Miedes, E., and Molina, A. 2018. Plant cell wallmediated immunity: Cell wall changes trigger disease resistance responses. Plant J. 93:614-636.

Baetsen-Young, A., Man Wai, C., VanBuren, R., and Day, B. 2020. Fusarium virguliforme transcriptional plasticity is revealed by host colonization of maize vs. soybean. Plant Cell 32:336-351.

Bankevich, A., Nurk, S., Antipov, D., Gurevich, A. A., Dvorkin, M., Kulikov, A. S., Lesin, V. M., Nikolenko, S. I., Pham, S., Prjibelski, A. D., Pyshkin, A. V., Sirotkin, A. V., Vyahhi, N., Tesler, G., Alekseyev, M. A., and Pevzner, P. A. 2012. SPAdes: A new genome assembly algorithm and its applications to single-cell sequencing. J. Comput. Biol. 19: 455-477.

Bednarek, P., Pislewska-Bednarek, M., Svatos, A., Schneider, B., Doubsky, J., Mansurova, M., Humphry, M., Consonni, C., Panstruga, R., SanchezVallet, A., Molina, A., and Schulze-Lefert, P. 2009. A glucosinolate metabolism pathway in living plant cells mediates broad-spectrum antifungal defense. Science 323:101-106.

Berrocal-Lobo, M., Molina, A., and Solano, R. 2002. Constitutive expression of ETHYLENE-RESPONSE-FACTOR1 in Arabidopsis confers resistance to several necrotrophic fungi. Plant J. 29:23-32.

Biely, P. 2012. Microbial carbohydrate esterases deacetylating plant polysaccharides. Biotechnol. Adv. 30:1575-1588.

Bindea, G., Mlecnik, B., Hackl, H., Charoentong, P., Tosolini, M., Kirilovsky, A., Fridman, W. H., Pagès, F., Trajanoski, Z., and Galon, J. 2009. ClueGO: A Cytoscape plug-in to decipher functionally grouped gene ontology and pathway annotation networks. Bioinformatics 25: 1091-1093.

Bolton, M. D., van Esse, H. P., Vossen, J. H., de Jonge, R., Stergiopoulos, I., Stulemeijer, I. J. E., van den Berg, G. C. M., Borrás-Hidalgo, O., Dekker, H. L., de Koster, C. G., de Wit, P. J. G. M., Joosten, M. H. A. J., and Thomma, B. P. H. J. 2008. The novel Cladosporium fulvum lysin motif effector Ecp6 is a virulence factor with orthologues in other fungal species. Mol. Microbiol. 69:119-136.

Brader, G., Compant, S., Vescio, K., Mitter, B., Trognitz, F., Ma, L.-J., and Sessitsch, A. 2017. Ecology and genomic insights into plantpathogenic and plant-nonpathogenic endophytes. Annu. Rev. Phytopathol. 55:61-83.

Bryant, D. M., Johnson, K., DiTommaso, T., Tickle, T., Couger, M. B., Payzin-Dogru, D., Lee, T. J., Leigh, N. D., Kuo, T. H., Davis, F. G., Bateman, J., Bryant, S., Guzikowski, A. R., Tsai, S. L., Coyne, S., Ye, W. W., Freeman, R. M., Jr., Peshkin, L., Tabin, C. J., Regev, A., Haas, B. J., and Whited, J. L. 2017. A tissue-mapped axolotl de novo transcriptome enables identification of limb regeneration factors. Cell Rep. 18:762-776.

Buchfink, B., Xie, C., and Huson, D. H. 2015. Fast and sensitive protein alignment using DIAMOND. Nat. Methods 12:59-60.

Buxdorf, K., Rahat, I., Gafni, A., and Levy, M. 2013. The epiphytic fungus Pseudozyma aphidis induces jasmonic acid- and salicylic acid/nonexpressor of PR1-independent local and systemic resistance. Plant Physiol. 161:2014-2022.

Cantarel, B. L., Korf, I., Robb, S. M. C., Parra, G., Ross, E., Moore, B., Holt, C., Sánchez Alvarado, A., and Yandell, M. 2008. MAKER: An easy-to-use annotation pipeline designed for emerging model organism genomes. Genome Res. 18:188-196.

Capella-Gutiérrez, S., Silla-Martínez, J. M., and Gabaldón, T. 2009. trimAl: A tool for automated alignment trimming in large-scale phylogenetic analyses. Bioinformatics 25:1972-1973. 
Carlucci, A., Raimondo, M. L., Santos, J., and Phillips, A. J. L. 2012. Plectosphaerella species associated with root and collar rots of horticultural crops in southern Italy. Persoonia 28:34-48.

Chang, H. X., Yendrek, C. R., Caetano-Anolles, G., and Hartman, G. L. 2016. Genomic characterization of plant cell wall degrading enzymes and in silico analysis of xylanases and polygalacturonases of Fusarium virguliforme. BMC Microbiol. 16:147.

Conesa, A., Götz, S., García-Gómez, J. M., Terol, J., Talón, M., and Robles, M. 2005. Blast2GO: a universal tool for annotation, visualization and analysis in functional genomics research. Bioinformatics 21:3674-3676.

Cortázar, A. R., Aransay, A. M., Alfaro, M., Oguiza, J. A., and Lavín, J. L. 2014. SECRETOOL: Integrated secretome analysis tool for fungi. Amino Acids 46:471-473.

D’Amico, M., Frisullo, S., and Cirulli, M. 2008. Endophytic fungi occurring in fennel, lettuce, chicory, and celery-Commercial crops in southern Italy. Mycol. Res. 112:100-107.

de Jonge, R., and Thomma, B. P. 2009. Fungal LysM effectors: Extinguishers of host immunity? Trends Microbiol. 17:151-157.

Delgado-Cerezo, M., Sánchez-Rodríguez, C., Escudero, V., Miedes, E., Fernández, P. V., Jordá, L., Hernández-Blanco, C., Sánchez-Vallet, A., Bednarek, P., Schulze-Lefert, P., Somerville, S., Estevez, J. M., Persson, S., and Molina, A. 2012. Arabidopsis heterotrimeric G-protein regulates cell wall defense and resistance to necrotrophic fungi. Mol. Plant 5: 98-114.

Dickman, M. B., and Mitra, A. 1992. Arabidopsis thaliana as a model for studying Sclerotinia sclerotiorum pathogenesis. Physiol. Mol. Plant Pathol. 41:255-263.

Dillard, H. R., Cobb, A. C., Shah, D. A., and Straight, K. E. 2005 Identification and characterization of russet on snap beans caused by Plectosporium tabacinum. Plant Dis. 89:700-704.

Douaiher, M. N., Nowak, E., Durand, R., Halama, P., and Reignault, P. 2007. Correlative analysis of Mycosphaerella graminicola pathogenicity and cell wall-degrading enzymes produced in vitro: The importance of xylanase and polygalacturonase. Plant Pathol. 56:79-86.

Durán, P., Thiergart, T., Garrido-Oter, R., Agler, M., Kemen, E., SchulzeLefert, P., and Hacquard, S. 2018. Microbial interkingdom interactions in roots promote Arabidopsis survival. Cell 175:P973-983.e14.

Edgar, R. C. 2004. MUSCLE: Multiple sequence alignment with high accuracy and high throughput. Nucleic Acids Res. 32:1792-1797.

Emms, D. M., and Kelly, S. 2015. OrthoFinder: Solving fundamental biases in whole genome comparisons dramatically improves orthogroup inference accuracy. Genome Biol. 16:157.

Emms, D. M., and Kelly, S. 2018. STAG: Species tree inference from all genes. bioRxiv 267914

Escudero, V., Jordá, L., Sopeña-Torres, S., Mélida, H., Miedes, E., MuñozBarrios, A., Swami, S., Alexander, D., McKee, L. S., Sánchez-Vallet, A., Bulone, V., Jones, A. M., and Molina, A. 2017. Alteration of cell wall xylan acetylation triggers defense responses that counterbalance the immune deficiencies of plants impaired in the $\beta$-subunit of the heterotrimeric G-protein. Plant J. 92:386-399.

Fesel, P. H., and Zuccaro, A. 2016. Dissecting endophytic lifestyle along the parasitism/mutualism continuum in Arabidopsis. Curr. Opin. Microbiol. 32:103-112.

García, E., Alonso, A., Platas, G., and Sacristan, S. 2013. The endophytic mycobiota of Arabidopsis thaliana. Fungal Divers. 60:71-89.

Gascuel, O. 1997. BIONJ: An improved version of the NJ algorithm based on a simple model of sequence data. Mol. Biol. Evol. 14: 685-695.

Gazis, R., Kuo, A., Riley, R., LaButti, K., Lipzen, A., Lin, J., Amirebrahimi, M., Hesse, C. N., Spatafora, J. W., Henrissat, B., Hainaut, M., Grigoriev, I. V., and Hibbett, D. S. 2016. The genome of Xylona heveae provides a window into fungal endophytism. Fungal Biol. 120:26-42.

Ge, X. T., and Barbetti, M. J. 2019. Host response of Arabidopsis thaliana ecotypes is determined by Sclerotinia sclerotiorum isolate type. Eur. J. Plant Pathol. 153:583-597.

Giraldo, A., and Crous, P. W. 2019. Inside Plectosphaerellaceae. Stud Mycol. 92:227-286.

Götz, M., Nirenberg, H., Krause, S., Wolters, H., Draeger, S., Buchner, A., Lottmann, J., Berg, G., and Smalla, K. 2006. Fungal endophytes in potato roots studied by traditional isolation and cultivation-independent DNA-based methods. FEMS Microbiol. Ecol. 58:404-413.

Guindon, S., Dufayard, J. F., Lefort, V., Anisimova, M., Hordijk, W., and Gascuel, O. 2010. New algorithms and methods to estimate maximumlikelihood phylogenies: Assessing the performance of PhyML 3.0. Syst. Biol. 59:307-321.

Gurevich, A., Saveliev, V., Vyahhi, N., and Tesler, G. 2013. QUAST: Quality assessment tool for genome assemblies. Bioinformatics 29: 1072-1075.
Guyon, K., Balagué, C., Roby, D., and Raffaele, S. 2014. Secretome analysis reveals effector candidates associated with broad host range necrotrophy in the fungal plant pathogen Sclerotinia sclerotiorum. BMC Genomics 15:336.

Hacquard, S., Kracher, B., Hiruma, K., Münch, P. C., Garrido-Oter, R., Thon, M. R., Weimann, A., Damm, U., Dallery, J.-F., Hainaut, M. Henrissat, B., Lespinet, O., Sacristán, S., Ver Loren van Themaat, E. Kemen, E., McHardy, A. C., Schulze-Lefert, P., and O'Connell, R. J. 2016. Survival trade-offs in plant roots during colonization by closely related beneficial and pathogenic fungi. Nat. Commun. 7:11362.

Hardoim, P. R., van Overbeek, L. S., Berg, G., Pirttilä, A. M., Compant, S., Campisano, A., Döring, M., and Sessitsch, A. 2015. The hidden world within plants: Ecological and evolutionary considerations for defining functioning of microbial endophytes. Microbiol. Mol. Biol. Rev. 79: 293-320.

Hernández-Blanco, C., Feng, D. X., Hu, J., Sánchez-Vallet, A., Deslandes, L., Llorente, F., Berrocal-Lobo, M., Keller, H., Barlet, X., Sánchez-Rodríguez, C., Anderson, L. K., Somerville, S., Marco, Y., and Molina, A. 2007. Impairment of cellulose synthases required for Arabidopsis secondary cell wall formation enhances disease resistance. Plant Cell 19:890-903.

Hiruma, K., Gerlach, N., Sacristán, S., Nakano, R. T., Hacquard, S., Kracher, B., Neumann, U., Ramírez, D., Bucher, M., O’Connell, R. J., and Schulze-Lefert, P. 2016. Root endophyte Colletotrichum tofieldiae confers plant fitness benefits that are phosphate status dependent. Cell 165:464-474

Hoff, K. J., Lange, S., Lomsadze, A., Borodovsky, M., and Stanke, M. 2016 BRAKER1: Unsupervised RNA-Seq-based genome annotation with GeneMark-ET and AUGUSTUS. Bioinformatics 32:767-769.

Hongsanan, S., Sánchez-Ramírez, S., Crous, P. W., Ariyawansa, H. A. Zhao, R. L., and Hyde, K. D. 2016. The evolution of fungal epiphytes Mycosphere 7:1690-1712.

Horbach, R., Navarro-Quesada, A. R., Knogge, W., and Deising, H. B. 2011. When and how to kill a plant cell: Infection strategies of plant pathogenic fungi. J. Plant Physiol. 168:51-62.

Huerta-Cepas, J., Capella-Gutiérrez, S., Pryszcz, L. P., Marcet-Houben, M., and Gabaldón, T. 2014. PhylomeDB v4: Zooming into the plurality of evolutionary histories of a genome. Nucleic Acids Res. 42:D897-D902.

Jimenez, P., and Zitter, T. A. 2005. First report of Plectosporium blight on pumpkin and squash caused by Plectosporium tabacinum in New York. Plant Dis. 89:432.

Jordá, L., Sopeña-Torres, S., Escudero, V., Nuñez-Corcuera, B., DelgadoCerezo, M., Torii, K. U., and Molina, A. 2016. ERECTA and BAK receptor like kinases interact to regulate immune responses in Arabidopsis. Front. Plant Sci. 7:897

Junker, C., Draeger, S., and Schulze, B. 2012. A fine line-endophytes or pathogens in Arabidopsis thaliana. Fungal Ecol. 5:657-662.

Kameshwar, A. K. S., Pereira Ramos, L., and Qin, W. 2019. CAZymesbased ranking of fungi (CBRF): An interactive web database for identifying fungi with extrinsic plant biomass degrading abilities. Bioresour. Bioprocess. 6:51.

Kamoun, S. 2009. The secretome of plant-associated fungi and oomycetes. Plant Relationships. The Mycota (A Comprehensive Treatise on Fungi as Experimental Systems for Basic and Applied Research). H. B. Deising, ed. Vol. 5. Springer, Berlin.

Katoh, K., Misawa, K., Kuma, K., and Miyata, T. 2002. MAFFT: A novel method for rapid multiple sequence alignment based on fast Fourier transform. Nucleic Acids Res. 30:3059-3066.

Kikot, G. E., Hours, R. A., and Alconada, T. M. 2009. Contribution of cell wall degrading enzymes to pathogenesis of Fusarium graminearum: A review. J. Basic Microbiol. 49:231-241.

Kim, K.-T., Jeon, J., Choi, J., Cheong, K., Song, H., Choi, G., Kang, S., and Lee, Y.-H. 2016. Kingdom-wide analysis of fungal small secreted proteins (SSPs) reveals their potential role in host association. Front. Plant Sci. 7:186.

Knapp, D. G., Németh, J. B., Barry, K., Hainaut, M., Henrissat, B., Johnson, J., Kuo, A., Lim, J. H. P., Lipzen, A., Nolan, M., Ohm, R. A., Tamás, L., Grigoriev, I. V., Spatafora, J. W., Nagy, L. G., and Kovács, G. M. 2018 Comparative genomics provides insights into the lifestyle and reveals functional heterogeneity of dark septate endophytic fungi. Sci. Rep. 8:6321.

Koch, E., and Slusarenko, A. 1990. Arabidopsis is susceptible to infection by a downy mildew fungus. Plant Cell 2:437-445.

Korf, I. 2004. Gene finding in novel genomes. BMC Bioinformatics 5:59.

Kumar, S., Stecher, G., Li, M., Knyaz, C., and Tamura, K. 2018. MEGA X: Molecular Evolutionary Genetics Analysis across computing platforms. Mol. Biol. Evol. 35:1547-1549.

Kurtz, S., Phillippy, A., Delcher, A. L., Smoot, M., Shumway, M., Antonescu, C., and Salzberg, S. L. 2004. Versatile and open software for comparing large genomes. Genome Biol. 5:R12. 
Laluk, K., and Mengiste, T. 2010. Necrotroph attacks on plants: Wanton destruction or covert extortion? Arabidopsis Book 8:e0136.

Leveau, J. 2015. Life of microbes on aerial plant parts. Principles of PlantMicrobe Interactions. B. Lugtenberg, ed. Springer, Cham, Switzerland.

Li, H. 2013. Aligning sequence reads, clone sequences and assembly contigs with BWA-MEM. ArXiv:1303.3997.

Li, H., Handsaker, B., Wysoker, A., Fennell, T., Ruan, J., Homer, N., Marth, G., Abecasis, G., Durbin, R. 2009. The sequence alignment/map (SAM) format and SAMtools. Bioinformatics 25:2078-2079.

Lindow, S. E., and Brandl, M. T. 2003. Microbiology of the phyllosphere. Appl. Environ. Microbiol. 69:1875-1883.

Lipka, V., Dittgen, J., Bednarek, P., Bhat, R., Wiermer, M., Stein, M., Landtag, J., Brandt, W., Rosahl, S., Scheel, D., Llorente, F., Molina, A., Parker, J., Somerville, S., and Schulze-Lefert, P. 2005. Pre- and postinvasion defenses both contribute to nonhost resistance in Arabidopsis. Science 310:1180-1183.

Llorente, F., Alonso-Blanco, C., Sánchez-Rodriguez, C., Jordá, L., and Molina, A. 2005. ERECTA receptor-like kinase and heterotrimeric G protein from Arabidopsis are required for resistance to the necrotrophic fungus Plectosphaerella cucumerina. Plant J. 43:165-180.

Llorente, F., Muskett, P., Sánchez-Vallet, A., López, G., Ramos, B., Sánchez-Rodríguez, C., Jordá, L., Parker, J., and Molina, A. 2008. Repression of the auxin response pathway increases Arabidopsis susceptibility to necrotrophic fungi. Mol. Plant 1:496-509.

Lombard, V., Golaconda Ramulu, H., Drula, E., Coutinho, P. M., and Henrissat, B. 2014. The carbohydrate-active enzymes database (CAZy) in 2013. Nucleic Acids Res. 42:D490-D495.

Lugtenberg, B., Caradus, J., and Johnson, L. 2016. Fungal endophytes for sustainable crop production. FEMS Microbiol. Ecol. 92:fiw194.

Lyu, X., Shen, C., Fu, Y., Xie, J., Jiang, D., Li, G., and Cheng, J. 2015. Comparative genomic and transcriptional analyses of the carbohydrateactive enzymes and secretomes of phytopathogenic fungi reveal their significant roles during infection and development. Sci. Rep. 5:15565.

Lyu, X., Shen, C., Fu, Y., Xie, J., Jiang, D., Li, G., and Cheng, J. 2016. A small secreted virulence-related protein is essential for the necrotrophic interactions of Sclerotinia sclerotiorum with its host plants. PLoS Pathog. 12:e1005435.

Malcolm, G. M., Kuldau, G. A., Gugino, B. K., and del Mar JiménezGasco, M. 2013. Hidden host plant associations of soilborne fungal pathogens: An ecological perspective. Phytopathology 103:538-544.

McKenna, A., Hanna, M., Banks, E., Sivachenko, A., Cibulskis, K., Kernytsky, A., Garimella, K., Altshuler, D., Gabriel, S., Daly, M., and DePristo, M. A. 2010. The Genome Analysis Toolkit: A MapReduce framework for analyzing next-generation DNA sequencing data. Genome Res. 20:1297-1303.

Medema, M. H., Blin, K., Cimermancic, P., de Jager, V., Zakrzewski, P., Fischbach, M. A., Weber, T., Takano, E., and Breitling, R. 2011. antiSMASH: Rapid identification, annotation and analysis of secondary metabolite biosynthesis gene clusters in bacterial and fungal genome sequences. Nucleic Acids Res. 39:W339-W346.

Mélida, H., Sopeña-Torres, S., Bacete, L., Garrido-Arandia, M., Jordá, L., López, G., Muñoz-Barrios, A., Pacios, L. F., and Molina, A. 2018. Nonbranched $\beta$-1,3-glucan oligosaccharides trigger immune responses in Arabidopsis. Plant J. 93:34-49.

Mine, A., Seyfferth, C., Kracher, B., Berens, M. L., Becker, D., and Tsuda, K. 2018. The defense phytohormone signaling network enables rapid, high-amplitude transcriptional reprogramming during effector-triggered immunity. Plant Cell 30:1199-1219.

Morales, J., Kadota, Y., Zipfel, C., Molina, A., and Torres, M. A. 2016. The Arabidopsis NADPH oxidases RbohD and RbohF display differential expression patterns and contributions during plant immunity. J. Exp. Bot. 67:1663-1676.

Newton, A. C., Fitt, B. D. L., Atkins, S. D., Walters, D. R., and Daniell, T. J. 2010. Pathogenesis, parasitism and mutualism in the trophic space of microbe-plant interactions. Trends Microbiol. 18:365-373.

Oliver, R. P., and Ipcho, S. V. 2004. Arabidopsis pathology breathes new life into the necrotrophs-vs.-biotrophs classification of fungal pathogens. Mol. Plant Pathol. 5:347-352.

Park, B. H., Karpinets, T. V., Syed, M. H., Leuze, M. R., and Uberbacher, E. C. 2010. CAZymes analysis toolkit (CAT): Web service for searching and analyzing carbohydrate-active enzymes in a newly sequenced organism using CAZy database. Glycobiology 20:1574-1584.

Pastorczyk, M., Kosaka, A., Piślewska-Bednarek, M., López, G., Frerigmann, H., Kułak, K., Glawischnig, E., Molina, A., Takano, Y., and Bednarek, P. 2020. The role of CYP71A12 monooxygenase in pathogen-triggered tryptophan metabolism and Arabidopsis immunity. New Phytol. 225:400-412.
Pawar, P. M.-A., Derba-Maceluch, M., Chong, S.-L., Gómez, L. D., Miedes, E., Banasiak, A., Ratke, C., Gaertner, C., Mouille, G., McQueen-Mason, S. J., Molina, A., Sellstedt, A., Tenkanen, M., and Mellerowicz, E. J. 2016. Expression of fungal acetyl xylan esterase in Arabidopsis thaliana improves saccharification of stem lignocellulose. Plant Biotechnol. J. 14 387-397.

Plett, J. M., and Martin, F. M. 2018. Know your enemy, embrace your friend: Using omics to understand how plants respond differently to pathogenic and mutualistic microorganisms. Plant J. 93:729-746.

Rai, M., and Agarkar, G. 2016. Plant-fungal interactions: What triggers the fungi to switch among lifestyles? Crit. Rev. Microbiol. 42:428-438.

Ramos, B., Alves-Santos, F. M., García-Sánchez, M. A., Martín-Rodrigues, N., Eslava, A. P., and Díaz-Mínguez, J. M. 2007. The gene coding for a new transcription factor (ftf1) of Fusarium oxysporum is only expressed during infection of common bean. Fungal Genet. Biol. 44:864-876.

Ramos, B., González-Melendi, P., Sánchez-Vallet, A., Sánchez-Rodríguez, C., López, G., and Molina, A. 2013. Functional genomics tools to decipher the pathogenicity mechanisms of the necrotrophic fungus Plectosphaerella cucumerina in Arabidopsis thaliana. Mol. Plant Pathol. 14:44-57.

Rastogi, G., Coaker, G. L., and Leveau, J. H. J. 2013. New insights into the structure and function of phyllosphere microbiota through highthroughput molecular approaches. FEMS Microbiol. Lett. 348:1-10.

Rawlings, N. D., Barrett, A. J., and Finn, R. 2016. Twenty years of the MEROPS database of proteolytic enzymes, their substrates and inhibitors. Nucleic Acids Res. 44 (D1):D343-D350.

Rieu, I., and Powers, S. J. 2009. Real-time quantitative RT-PCR: Design, calculations, and statistics. Plant Cell 21:1031-1033.

Saier, M. H., Jr., Reddy, V. S., Tamang, D. G., and Västermark, A. 2014 The transporter classification database. Nucleic Acids Res. 42: D251-D258.

Sánchez-Rodríguez, C., Estévez, J. M., Llorente, F., Hernández-Blanco, C. Jordá, L., Pagán, I., Berrocal, M., Marco, Y., Somerville, S., and Molina, A. 2009. The ERECTA receptor-like kinase regulates cell wallmediated resistance to pathogens in Arabidopsis thaliana. Mol. Plant-Microbe Interact. 22:953-963.

Sánchez-Vallet, A., López, G., Ramos, B., Delgado-Cerezo, M., Riviere, M.-P., Llorente, F., Fernández, P. V., Miedes, E., Estevez, J. M., Grant M., and Molina, A. 2012. Disruption of abscisic acid signaling constitutively activates Arabidopsis resistance to the necrotrophic fungus Plectosphaerella cucumerina. Plant Physiol. 160:2109-2124.

Sánchez-Vallet, A., Ramos, B., Bednarek, P., López, G., PiślewskaBednarek, M., Schulze-Lefert, P., and Molina, A. 2010. Tryptophanderived secondary metabolites in Arabidopsis thaliana confer non-host resistance to necrotrophic Plectosphaerella cucumerina fungi. Plant J. 63:115-127.

Schulz, B., and Boyle, C. 2005. The endophytic continuum. Mycol. Res. 109:661-686

Seidl, M. F., Faino, L., Shi-Kunne, X., van den Berg, G. C. M., Bolton, M. D., and Thomma, B. P. H. J. 2015. The genome of the saprophytic fungus Verticillium tricorpus reveals a complex effector repertoire resembling that of its pathogenic relatives. Mol. Plant-Microbe Interact. 28:362-373.

Selosse, M.-A., Schneider-Maunoury, L., and Martos, F. 2018. Time to rethink fungal ecology? Fungal ecological niches are often prejudged. New Phytol. 217:968-972.

Simão, F. A., Waterhouse, R. M., Ioannidis, P., Kriventseva, E. V., and Zdobnov, E. M. 2015. BUSCO: Assessing genome assembly and annotation completeness with single-copy orthologs. Bioinformatics 31: 3210-3212.

Spanu, P. D., and Panstruga, R. 2017. Editorial: Biotrophic plant-microbe interactions. Front. Plant Sci. 8:192.

Stanke, M., Keller, O., Gunduz, I., Hayes, A., Waack, S., and Morgenstern, B. 2006. AUGUSTUS: Ab initio prediction of alternative transcripts. Nucleic Acids Res. 34:W435-W439.

Stein, M., Dittgen, J., Sánchez-Rodríguez, C., Hou, B. H., Molina, A., Schulze-Lefert, P., Lipka, V., and Somerville, S. 2006. Arabidopsis PEN3/PDR8, an ATP binding cassette transporter, contributes to nonhost resistance to inappropriate pathogens that enter by direct penetration. Plant Cell 18:731-746.

Stergiopoulos, I., and de Wit, P. J. 2009. Fungal effector proteins. Annu. Rev. Phytopathol. 47:233-263.

Su, L., Deng, H., and Niu, Y.-C. 2017. Phylogenetic analysis of Plectosphaerella species based on multi-locus DNA sequences and description of P. sinensis sp. nov. Mycol. Prog. 16:823-829.

Subramanian, A. R., Kaufmann, M., and Morgenstern, B. 2008. DIALIGNTX: Greedy and progressive approaches for segment-based multiple sequence alignment. Algorithms Mol. Biol. 3:6. 
Takashima, M., Manabe, R.-I., and Ohkuma, M. 2019. Draft genome sequences of basidiomycetous epiphytic phylloplane yeast type strains Dioszegia crocea JCM 2961 and Dioszegia aurantiaca JCM 2956. Microbiol. Resour. Announc. 8:e01727-18.

Tamura, K., and Nei, M. 1993. Estimation of the number of nucleotide substitutions in the control region of mitochondrial DNA in humans and chimpanzees. Mol. Biol. Evol. 10:512-526.

Thiergart, T., Durán, P., Ellis, T., Vannier, N., Garrido-Oter, R., Kemen, E., Roux, F., Alonso-Blanco, C., Ågren, J., Schulze-Lefert, P., and Hacquard, S. 2020. Root microbiota assembly and adaptive differentiation among European Arabidopsis populations. Nat. Ecol. Evol. 4: 122-131.

Tierens, K. F., Thomma, B. P., Brouwer, M., Schmidt, J., Kistner, K., Porzel, A., Mauch-Mani, B., Cammue, B. P., and Broekaert, W. F. 2001. Study of the role of antimicrobial glucosinolate-derived isothiocyanates in resistance of Arabidopsis to microbial pathogens. Plant Physiol. 125: 1688-1699.

Ton, J., and Mauch-Mani, B. 2004. Beta-amino-butyric acid-induced resistance against necrotrophic pathogens is based on ABA-dependent priming for callose. Plant J. 38:119-130.

Torres, M. A., Dangl, J. L., and Jones, J. D. G. 2002. Arabidopsis gp91 ${ }^{\text {phox }}$ homologues AtrbohD and AtrbohF are required for accumulation of reactive oxygen intermediates in the plant defense response. Proc. Natl. Acad. Sci. U.S.A. 99:517-522.

Trapnell, C., Roberts, A., Goff, L., Pertea, G., Kim, D., Kelley, D. R., Pimentel, H., Salzberg, S. L., Rinn, J. L., and Pachter, L. 2012. Differential gene and transcript expression analysis of RNA-seq experiments with TopHat and Cufflinks. Nat. Protoc. 7:562-578.

Tsuda, K., Sato, M., Stoddard, T., Glazebrook, J., and Katagiri, F. 2009. Network properties of robust immunity in plants. PLoS Genet. 5: e1000772.

Usami, T., and Katagiri, H. 2017. Pathogenicity of Plectosphaerella species on lettuce and susceptibility of lettuce cultivars. J. Gen. Plant Pathol. 83: 366-372.

Vitale, S., Maccaroni, M., and Belisario, A. 2007. First report of zucchini collapse by Fusarium solani f. sp. cucurbitae race 1 and Plectosporium tabacinum in Italy. Plant Dis. 91:325.

Vorholt, J. A. 2012. Microbial life in the phyllosphere. Nat. Rev. Microbiol. 10:828-840

Wallace, I. M., O’Sullivan, O., Higgins, D. G., and Notredame, C. 2006. MCoffee: Combining multiple sequence alignment methods with T-Coffee. Nucleic Acids Res. 34:1692-1699.

Wan, J., Zhang, X. C., Neece, D., Ramonell, K. M., Clough, S., Kim, S. Y., Stacey, M. G., and Stacey, G. 2008. A LysM receptor-like kinase plays a critical role in chitin signaling and fungal resistance in Arabidopsis. Plant Cell 20:471-481.

Wang, B., Liang, X., Gleason, M. L., Zhang, R., and Sun, G. 2017. Genome sequence of the ectophytic fungus Ramichloridium luteum reveals unique evolutionary adaptations to plant surface niche. BMC Genomics 18:729.

Wang, M., Liu, B., Ruan, R., Zeng, Y., Luo, J., and Li, H. 2020. Genomic sequencing of Phyllosticta citriasiana provides insight into its conservation and diversification with two closely related Phyllosticta species associated with Citrus. Front. Microbiol. 10:2979.

Wang, X., Jiang, N., Liu, J., Liu, W., and Wang, G. L. 2014. The role of effectors and host immunity in plant-necrotrophic fungal interactions. Virulence 5:722-732.
Wang, X., Zhang, X., Liu, L., Xiang, M., Wang, W., Sun, X., Che, Y., Guo, L., Liu, G., Guo, L., Wang, C., Yin, W. B., Stadler, M., Zhang, X., and Liu, X. 2015. Genomic and transcriptomic analysis of the endophytic fungus Pestalotiopsis fici reveals its lifestyle and high potential for synthesis of natural products. BMC Genomics 16:28.

Whipps, J. M., Hand, P., Pink, D., and Bending, G. D. 2008. Phyllosphere microbiology with special reference to diversity and plant genotype. J. Appl. Microbiol. 105:1744-1755.

Windram, O., Madhou, P., McHattie, S., Hill, C., Hickman, R., Cooke, E., Jenkins, D. J., Penfold, C. A., Baxter, L., Breeze, E., Kiddle, S. J., Rhodes, J., Atwell, S., Kliebenstein, D. J., Kim, Y. S., Stegle, O. Borgwardt, K., Zhang, C., Tabrett, A., Legaie, R., Moore, J., Finkenstadt, B., Wild, D. L., Mead, A., Rand, D., Beynon, J., Ott, S., BuchananWollaston, V., and Denby, K. J. 2012. Arabidopsis defense against Botrytis cinerea: Chronology and regulation deciphered by highresolution temporal transcriptomic analysis. Plant Cell 24:3530-3557.

Xu, C., Chen, H., Gleason, M. L., Xu, J. R., Liu, H., Zhang, R., and Sun, G. 2016. Peltaster fructicola genome reveals evolution from an invasive phytopathogen to an ectophytic parasite. Sci. Rep. 6:22926.

Xu, X.-H., Su, Z.-Z., Wang, C., Kubicek, C. P., Feng, X.-X., Mao, L.-J., Wang, J.-Y., Chen, C., Lin, F.-C., and Zhang, C.-L. 2014. The rice endophyte Harpophora oryzae genome reveals evolution from a pathogen to a mutualistic endophyte. Sci. Rep. 4:5783.

Yao, H., Sun, X., He, C., Maitra, P., Li, X.-C., and Guo, L.-D. 2019. Phyllosphere epiphytic and endophytic fungal community and network structures differ in a tropical mangrove ecosystem. Microbiome 7:57.

Yu, Q., and Coosemans, J. 1998. Fungi associated with cysts of Globodera rostochiensis, G. pallida, and Heterodera schachtii; and egg masses and females of Meloidogyne hapla in Belgium. Phytoprotection 79:63-69.

Yuan, Y., Teng, Q., Zhong, R., and Ye, Z. H. 2013. The Arabidopsis DUF231 domain-containing protein ESK1 mediates 2-O- and 3-O-acetylation of xylosyl residues in xylan. Plant Cell Physiol. 54:1186-1199.

Zaharia, M., Bolosky, W. J., Curtis, K., Fox, A., Patterson, D., Shenker, S., Stoica, I., Karp, R. M., and Sittler, T. 2011. Faster and more accurate sequence alignment with SNAP. arXiv:1111.5572v1.

Zeilinger, S., Gupta, V. K., Dahms, T. E. S., Silva, R. N., Singh, H. B., Upadhyay, R. S., Gomes, E. V., Tsui, C. K., and Nayak S, C. 2016. Friends or foes? Emerging insights from fungal interactions with plants. FEMS Microbiol. Rev. 40:182-207.

Zerbino, D. R., and Birney, E. 2008. Velvet: Algorithms for de novo short read assembly using de Bruijn graphs. Genome Res. 18:821-829.

Zhang, Z., Schwartz, S., Wagner, L., and Miller, W. 2000. A greedy algorithm for aligning DNA sequences. J. Comput. Biol. 7:203-214.

Zhao, Y., Hull, A. K., Gupta, N. R., Goss, K. A., Alonso, J., Ecker, J. R., Normanly, J., Chory, J., and Celenza, J. L. 2002. Trp-dependent auxin biosynthesis in Arabidopsis: Involvement of cytochrome P450s CYP79B2 and CYP79B3. Genes Dev. 16:3100-3112.

Zhao, Z., Liu, H., Wang, C., and Xu, J.-R. 2013. Comparative analysis of fungal genomes reveals different plant cell wall degrading capacity in fungi. BMC Genomics 14:274

Zhou, J., Bi, S., Chen, H., Chen, T., Yang, R., Li, M., Fu, Y., and Jia, A.-Q. 2017. Anti-biofilm and antivirulence activities of metabolites from Plectosphaerella cucumerina against Pseudomonas aeruginosa. Front. Microbiol. 8:769. 